\title{
De copulis non est disputandum
}

\section{Copulae: An Overview}

\author{
Wolfgang Härdle* \\ Ostap Okhrin*
}

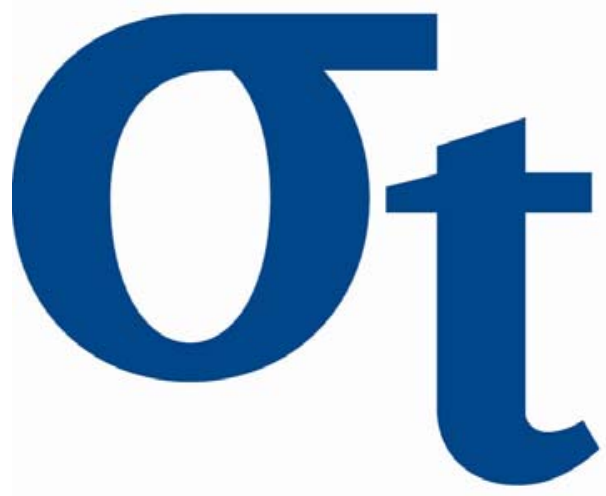

* Humboldt-Universität zu Berlin, Germany

This research was supported by the Deutsche Forschungsgemeinschaft through the SFB 649 "Economic Risk". 


\title{
De copulis non est disputandum*
}

\author{
Copulae: An Overview
}

\author{
Wolfgang Karl Härdle† Ostap Okhrin
}

May 27, 2009

\begin{abstract}
Normal distribution of the residuals is the traditional assumption in the classical multivariate time series models. Nevertheless it is not very often consistent with the real data. Copulae allows for an extension of the classical time series models to nonelliptically distributed residuals. In this paper we apply different copulae to the calculation of the static and dynamic Value-at-Risk of portfolio returns and Profit-and-Loss function. In our findings copula based multivariate model provide better results than those based on the normal distribution.
\end{abstract}

Keywords: copula; multivariate distribution; value-at-risk; multivariate dependence.

JEL Classification: C13, C14, C50.

\section{Introduction}

Understanding the joint distribution of high dimensional data is fundamental in applied statistics. The conventional procedure to model joint distributions is to approximate them with multivariate normal distributions.

That implies, however, that the dependence structures is reduced to a fixed type. Predetermining a multivariate normal distribution means that the tails of the distribution are not too heavy, the distribution is symmetric and that the dependence between variables is linear.

Empirical evidence for these assumptions are barely verified and an alternative model is needed, with more flexible dependence structure and arbitrary marginal distributions. These are exactly the characteristics of copulae.

Copulae are very useful for modelling and estimating multivariate distributions. The flexibilty of copulae basically follows from Sklar's Theorem, which says that each joint

*The financial support from the Deutsche Forschungsgemeinschaft via SFB 649 "Okonomisches Risiko", Humboldt-Universität zu Berlin is gratefully acknowledged.

†CASE - Center for Applied Statistics and Economics, Institute for Statistics and Econometrics of Humboldt-Universität zu Berlin, Spandauer Straße 1, 10178 Berlin, Germany. Email: haerdle@wiwi.huberlin.de.

${ }^{\ddagger}$ CASE - Center for Applied Statistics and Economics, Institute for Statistics and Econometrics of Humboldt-Universität zu Berlin, Spandauer Straße 1, 10178 Berlin, Germany. Email: ostap.okhrin@wiwi.hu-berlin.de 
distribution can be "decomposed" into its marginal distributions and a copula $C$ "responsible" for the dependence structure:

$$
F\left(x_{1} \ldots, x_{d}\right)=C\left\{F_{1}\left(x_{1}\right), \ldots, F_{d}\left(x_{d}\right)\right\}
$$

Two important factors for practical applications rely on this theorem:

1. The construction of multivariate distributions may be done in two independent steps: the specification of marginal distributions - not necessarily identical - and the specification of a dependence structure. Copulae "couple together" the marginal distributions into a multivariate distribution with the desired dependence structure.

2. Joint distributions can be separately estimated from a sample of observations: the marginal distributions are estimated first, the dependence structure later.

The copula approach gives us more freedom than the normality assumptions, marginal distributions with asymmetric heavy tails (typical for financial returns) can be combined with different dependence structures, resulting in multivariate distributions (far different from the multivariate normal) that better describe the empirical characteristics of financial returns distribution.

Moreover, copulae allow for dynamical modelling and adaption to portfolios, different copulae with distinct properties can be associated to different portfolios according to their specific dependence structures. Furthermore, copulae may change as time evolves, reflecting the evolution of the dependence between financial assets.

The structure of this paper is as follows. In the next section we give a short review of the copula theory. In the Section 3 we deals with different copula classes used in the calculation. The simulation and estimation techniques are provided in Sections 4 and 5 respectively. The first static problem on the calculation of the Value-at-Risk for the portfolio return has been discussed in Sections 6 and in the beginning of Section 7. Subsections 7.1 and 7.2 deals with the dynamic estimation of the Value-at-Risk for the Profit and Loss function. The paper is finished with summary.

\section{Copulae}

The description of copulae for measuring and modelling dependence with its main properties is the subject of this section. The term copula goes back to the works of Sklar (1959) were it was first mentioned. There are a lot of different equivalent definitions that could define the copula, but the most general is the following one.

Definition 1 (Copula) A d-dimensional copula is a d-dimensional distribution with all uniform marginal distributions.

Note that by considering random variables $X_{1}, \ldots, X_{d}$ with univariate distribution functions $F_{X_{1}}, \ldots, F_{X_{d}}$ and the random variables $U_{i}=F_{X_{i}}\left(X_{i}\right), i=1, \ldots, d$ uniformly distributed in $[0,1]$, a copula may be interpreted as the joint distribution of the marginal distributions. 
Copulae gained popularity through Sklar's (1959) work where the term was first coined. However, many results had already been proved by Hoeffding (1940) and Hoeffding (1941), who could have been the founder of a copula theory, if he had considered the stochastically more intuitive dependency over the unit cube $[0,1]^{2}$ rather than over $[-1 / 2,1 / 2]^{2}$ as he had done. Copulae allow marginal distributions to be separated from the dependency structure. Sklar's theorem connects copulae with distribution functions such that from the one side every distribution function can be "decomposed" into its marginal distribution and (at least) one copula and from the other side a (unique) copula is obtained from "decoupling" every (continuous) multivariate distribution function from its marginal distributions.

Theorem 1 (Sklar's theorem) Let $F$ be a multivariate distribution function with margins $F_{1}, \ldots, F_{d}$, then a copula $C$ exists such that

$$
F\left(x_{1}, \ldots, x_{d}\right)=C\left\{F_{1}\left(x_{1}\right), \ldots, F_{k}\left(x_{d}\right)\right\}, \quad x_{1}, \ldots, x_{d} \in \overline{\mathbb{R}} .
$$

If $F_{i}$ are continuous for $i=1, \ldots, d$ then $C$ is unique. Otherwise $C$ is uniquely determined on $F_{1}(\overline{\mathbb{R}}) \times \cdots \times F_{d}(\overline{\mathbb{R}})$.

Conversely, if $C$ is a copula and $F_{1}, \ldots, F_{d}$ are univariate distribution functions, then the function $F$ defined above is a multivariate distribution function with margins $F_{1}, \ldots, F_{d}$.

The representation in Sklar's Theorem can be used to construct new multivariate distributions by changing either the copula function or marginal distributions. For an arbitrary continuous multivariate distribution we can determine its copula from the transformation

$$
C\left(u_{1}, \ldots, u_{d}\right)=F\left\{F_{1}^{-1}\left(u_{1}\right), \ldots, F_{d}^{-1}\left(u_{d}\right)\right\}, \quad u_{1}, \ldots, u_{d} \in[0,1],
$$

where $F_{i}^{-1}$ are inverse marginal distribution functions.

Since the copula function is a multivariate distribution with uniform margins, it follows that the copula density can be determined in the usual way

$$
c\left(u_{1}, \ldots, u_{d}\right)=\frac{\partial^{d} C\left(u_{1}, \ldots, u_{d}\right)}{\partial u_{1} \ldots \partial u_{d}}, \quad u_{1}, \ldots, u_{d} \in[0,1],
$$

Being armed with Theorem 1 and (??) we can write the density function $f(\cdot)$ of the $d$-variate distribution $F$ in terms of copula as follows

$$
f\left(x_{1}, \ldots, x_{d}\right)=c\left\{F_{1}\left(x_{1}\right), \ldots, F_{d}\left(x_{d}\right)\right\} \prod_{i=1}^{d} f_{i}\left(x_{i}\right), \quad x_{1}, \ldots, x_{d} \in \overline{\mathbb{R}} .
$$

A detailed discussion with proofs and deep mathematical treatment can be found in Joe (1997) and Nelsen (2006). A practical introduction is given in Deutsch and Eller (1999). Embrechts, McNeil and Straumann (1999b) discuss restrictions of the copula technique and their relation to the classical correlation analysis.

\section{Copula Classes}

Since there are plenty of functions satisfying the assumption of Theorem 1 they should be classified by construction and properties. Here we consider several main classes, like simplest, elliptical, Archimedean copulae and hierarchical Archimedean copulae. 


\subsection{Simplest Copulae}

Special cases, like independence and perfect positive or negative dependence can be represented by copulae. If $d$ random variables $X_{1}, \ldots, X_{d}$ are stochastically independent from Theorem 1, then the structure of such a relationship is given by the product copula

$$
\Pi\left(u_{1}, \ldots, u_{d}\right)=\prod_{j=1}^{d} u_{j} .
$$

Copulae are bounded, this means that for all $u=\left(u_{1}, \ldots, u_{d}\right)^{\top} \in[0,1]^{d}$ :

$$
W\left(u_{1}, \ldots, u_{d}\right) \leq C\left(u_{1}, \ldots, u_{d}\right) \leq M\left(u_{1}, \ldots, u_{d}\right)
$$

where

$$
M\left(u_{1}, \ldots, u_{d}\right)=\min \left(u_{1}, \ldots, u_{d}\right)
$$

is called the Fréchet-Hoeffding lower bound and

$$
W\left(u_{1}, \ldots, u_{d}\right)=\max \left(\sum_{i=1}^{d} u_{i}-d+1,0\right)
$$

is the Fréchet-Hoeffding upper bound. While $M$ is not a copula for $d>2, W$ is a copula for all $d$. Both structures represent the perfect negative and perfect positive dependence. From this observation we may conclude that an arbitrary copula $C$ reflects dependence which lies between the perfect negative and positive one.

\subsection{Elliptical Copulae}

The elliptical copulae are derived from the elliptical distributions using Theorem 1 . In the bivariate case one has that a bivariate copula is elliptical if, and only if, it is equal to its associated copula

$$
\begin{aligned}
C\left(u_{1}, u_{2}, \theta\right) & =\bar{C}\left(u_{1}, u_{2}, \theta\right) \\
& =u_{1}+u_{2}-1+C\left(1-u_{1}, 1-u_{2}, \theta\right), \quad u_{1}, u_{2} \in[0,1] .
\end{aligned}
$$

The most prominent examples of elliptical copulae are Gaussian and $t$-copula.

\section{Gaussian Copula}

The Gaussian copula represents the dependence structure of the multivariate normal distribution, that means that normal marginal distributions are combined with a Gaussian copula to form multivariate normal distributions. The combination of non-normal marginal distributions with a Gaussian copula results in meta-Gaussian distributions, i.e., distributions where only the dependence structure is Gaussian. 
To obtain the Gaussian copula, let $X=\left(X, \ldots, X_{d}\right)^{\top} \sim \mathrm{N}_{d}(\mu, \Sigma)$ with $X_{j} \sim \mathrm{N}\left(\mu_{j}, \sigma_{j}\right)$ for $j=1, \ldots, d$. A copula $C$ exists:

$$
F\left(x_{1}, \ldots, x_{d}\right)=C\left\{F_{1}\left(x_{1}\right), \ldots, F_{d}\left(x_{d}\right)\right\}
$$

where $F_{j}$ is the distribution function of $X_{j}$ and $F$ the distribution function of $X$. Let $Y_{j}=T_{j}\left(X_{j}\right), T_{j}(x)=\left(x-\mu_{j}\right) / \sigma_{j}$. Then $Y_{j} \sim \mathrm{N}(0,1)$ and $Y=\left(Y_{1}, \ldots, Y_{d}\right)^{\top} \sim \mathrm{N}_{d}(0, \Psi)$ where $\Psi$ is the correlation matrix associated with $\Sigma$. A copula $C_{\Psi}^{G a}$, called Gaussian copula exists as follows:

$$
F_{Y}\left(y_{1}, \ldots, y_{d}\right)=C_{\Psi}^{G a}\left\{\Phi\left(y_{1}\right), \ldots, \Phi\left(y_{d}\right)\right\}
$$

An explicit expression for the Gaussian copula is obtained by rewriting (3) with $u_{j}=$ $\Phi\left(y_{j}\right)$ :

$$
\begin{aligned}
C_{\Psi}^{G a}\left(u_{1}, \ldots, u_{d}\right) & =F_{Y}\left\{\Phi^{-1}\left(u_{1}\right), \ldots, \Phi^{-1}\left(u_{d}\right)\right\} \\
\quad= & \int_{-\infty}^{\Phi^{-1}\left(u_{1}\right)} \cdots \int_{-\infty}^{\Phi^{-1}\left(u_{d}\right)}(2 \pi)^{-\frac{d}{2}}|\Psi|^{-\frac{1}{2}} \exp \left(-\frac{1}{2} r^{\top} \Psi^{-1} r\right) d r_{1} \ldots d r_{d} .
\end{aligned}
$$

The density of the Gaussian copula is given by

$$
c_{\Psi}^{G a}\left(u_{1}, \ldots, u_{d}\right)=|\Psi|^{-\frac{1}{2}} \exp \left\{-\frac{1}{2} \zeta^{\top}\left(\Psi^{-1}-I_{d}\right) \zeta\right\} .
$$

\section{Student's $t$-Copula}

The $t$-copula, containing the dependence structure from the multivariate $t$-distribution, may be obtained in a similar way.

Let $X=\left(X_{1}, \ldots, X_{d}\right)^{\top} \sim t_{d}(\nu, \mu, \Sigma)$ and $Y=\left(Y_{1}, \ldots, Y_{d}\right)^{\top} \sim t_{d}(\nu, 0, \Psi)$ where $\Psi$ is the correlation matrix associated with $\Sigma$. The unique copula from $Y$ is the Student's $t$-copula $C_{\nu, \Psi}^{t}$. For $u=\left(u_{1}, \ldots, u_{d}\right)^{\top} \in[0,1]^{d}$, the Student's t-copula is given by

$$
C_{\nu, \Psi}^{t}\left(u_{1}, \ldots, u_{d}\right)=t_{\nu, \Psi}\left\{t_{\nu}^{-1}\left(u_{1}\right), \ldots, t_{\nu}^{-1}\left(u_{d}\right)\right\}
$$

where $t_{\nu}^{-1}$ is the quantile function from the univariate $t$-distribution and $t_{\nu, \Psi}$ the distribution function of $Y$.

The density of the t-copula is given by

$$
\begin{aligned}
c_{\nu, \Psi}^{t}\left(u_{1}, \ldots, u_{d}\right) & =\frac{t_{\nu, \Psi}\left\{t_{\nu}^{-1}\left(u_{1}\right), \ldots, t_{\nu}^{-1}\left(u_{d}\right)\right\}}{\prod_{j=1}^{d} t_{\nu, \Psi}\left\{t_{\nu}^{-1}\left(u_{j}\right)\right\}} . \\
& =|\Psi|^{-\frac{1}{2}} \frac{\Gamma\left(\frac{\nu+d}{2}\right)\left\{\Gamma\left(\frac{\nu}{2}\right)\right\}^{d-1}\left(1+\frac{1}{\nu} \zeta^{\top} \Psi^{-1} \zeta\right)^{-\frac{\nu+d}{2}}}{\left\{\Gamma\left(\frac{\nu+1}{2}\right)\right\}^{d} \prod_{j=1}^{d}\left(1+\frac{1}{\nu} \zeta_{j}^{2}\right)^{-\frac{\nu+1}{2}}} .
\end{aligned}
$$




\subsection{Archimedean Copulae}

As opposed to elliptical copulae, Archimedean copulae are not constructed using Theorem 1. but are related to Laplace transforms of univariate distribution functions. Let $\mathbb{L}$ denote the class of Laplace transforms which consists of strictly decreasing differentiable functions Joe (1997), i.e.

$$
\mathbb{L}=\left\{\phi:[0 ; \infty) \rightarrow[0,1] \mid \phi(0)=1, \phi(\infty)=0 ;(-1)^{j} \phi^{(j)} \geq 0 ; j=1, \ldots, \infty\right\} .
$$

The function $C:[0,1]^{d} \rightarrow[0,1]$ defined as

$$
C\left(u_{1}, \ldots, u_{d}\right)=\phi\left\{\phi^{-1}\left(u_{1}\right)+\cdots+\phi^{-1}\left(u_{d}\right)\right\}, \quad u_{1}, \ldots, u_{d} \in[0,1]
$$

is a $d$-dimensional Archimedean copula, where $\phi \in \mathbb{L}$ and is called the generator of the copula. It is straightforward to show that $C\left(u_{1}, \ldots, u_{d}\right)$ satisfies the conditions of Definition 1 .

Some $d$-dimensional Archimedean copulae are presented below.

Frank (1979) copula, $0 \leq \theta<\infty$.

The first popular Archimedean copula is the so called Frank copula, which is the only elliptical Archimedean copula. Its generator and copula functions are

$$
\begin{aligned}
\phi(x, \theta) & =\theta^{-1} \log \left\{1-\left(1-e^{-\theta}\right) e^{-x}\right\}, \quad 0 \leq \theta<\infty, x \in[0, \infty) . \\
C_{\theta}\left(u_{1}, \ldots, u_{d}\right) & =-\frac{1}{\theta} \log \left[1+\frac{\prod_{j=1}^{d}\left\{\exp \left(-\theta u_{j}\right)-1\right\}}{\{\exp (-\theta)-1\}^{d-1}}\right] .
\end{aligned}
$$

The dependence becomes maximal when $\theta$ tends to infinity and independence is achieved when $\theta=0$.

Gumbel (1960) copula, $1 \leq \theta<\infty$.

The Gumbel copula is frequently used in financial applications. Its generator and copula functions are

$$
\begin{aligned}
\phi(x, \theta) & =\exp \left\{-x^{1 / \theta}\right\}, \quad 1 \leq \theta<\infty, x \in[0, \infty) \\
C_{\theta}\left(u_{1}, \ldots, u_{d}\right) & =\exp \left[-\left\{\sum_{j=1}^{d}\left(-\log u_{j}\right)^{\theta}\right\}^{\theta^{-1}}\right] .
\end{aligned}
$$

Consider a bivariate distribution based on the Gumbel copula with univariate extreme value marginal distributions. Genest and Rivest (1989) showed that this distribution is 
the only bivariate extreme value distribution based on an Archimedean copula. Moreover, all distributions based on Archimedean copulae belong to its domain of attraction under common regularity conditions. In contrary to the elliptical copulae, the Gumbel copula leads to asymmetric contour diagrams. The Gumbel copula shows stronger linkage between positive values, however, it also shows more variability and more mass in the negative tail.

For $\theta>1$ this copula allows for the generation of dependence in the upper tail. For $\theta \rightarrow 1$, the Gumbel copula reduces to the product copula and for $\theta \rightarrow \infty$ we obtain the Fréchet-Hoeffding upper bound.

Clayton (1978) copula, $-1 \leq \theta<\infty, \theta \neq 0$.

The Clayton copula which, in contrast to the Gumbel copula, has more mass on the lower tail, and less on the upper. The generator and copula function are

$$
\begin{aligned}
\phi(x, \theta) & =(\theta x+1)^{-\frac{1}{\theta}}, \quad-1 \leq \theta<\infty, \theta \neq 0, x \in[0, \infty), \\
C_{\theta}\left(u_{1}, \ldots, u_{d}\right) & =\left\{\left(\sum_{j=1}^{d} u_{j}^{-\theta}\right)-d+1\right\}^{-\theta^{-1}} .
\end{aligned}
$$

The Clayton copula is one of few copulae that has a simple explicit form of density for any dimension

$$
c_{\theta}\left(u_{1}, \ldots, u_{d}\right)=\prod_{j=1}^{d}\{1+(j-1) \theta\} u_{j}^{-(\theta+1)}\left(\sum_{j=1}^{d} u_{j}^{-\theta}-d+1\right)^{-\left(\theta^{-1}+d\right)} .
$$

As the parameter $\theta$ tends to infinity, dependence becomes maximal and as $\theta$ tends to zero, we have independence. As $\theta \rightarrow-1$, the distribution tends to the lower Fréchet bound.

\subsection{Hierarchical Archimedean Copulae}

A recently developed flexible method is provided by hierarchical Archimedean copulae (HAC). The special, so called fully nested case of the copula function is:

$$
\begin{aligned}
C\left(u_{1}, \ldots, u_{d}\right) & =\phi_{d-1}\left\{\phi _ { d - 1 } ^ { - 1 } \circ \phi _ { d - 2 } \left(\ldots \left[\phi_{2}^{-1} \circ \phi_{1}\left\{\phi_{1}^{-1}\left(u_{1}\right)+\phi_{1}^{-1}\left(u_{2}\right)\right\}\right.\right.\right. \\
& \left.\left.\left.+\phi_{2}^{-1}\left(u_{3}\right)\right]+\cdots+\phi_{d-2}^{-1}\left(u_{d-1}\right)\right)+\phi_{d-1}^{-1}\left(u_{d}\right)\right\} \\
& =\phi_{d-1}\left[\phi_{d-1}^{-1} \circ C\left(\left\{\phi_{1}, \ldots, \phi_{d-2}\right\}\right)\left(u_{1}, \ldots, u_{d-1}\right)+\phi_{d-1}^{-1}\left(u_{d}\right)\right]
\end{aligned}
$$

for $\phi_{d-i}^{-1} \circ \phi_{d-j} \in \mathbb{L}^{*}, i<j$, where

$$
\begin{aligned}
\mathbb{L}^{*}= & \{\omega:[0 ; \infty) \rightarrow[0, \infty) \mid \omega(0)=0, \\
& \left.\omega(\infty)=\infty ;(-1)^{j-1} \omega^{(j)} \geq 0 ; j=1, \ldots, \infty\right\} .
\end{aligned}
$$

In contrast to the Archimedean copula, the HAC defines the whole dependency structure in a recursive way. At the lowest level the dependency between the first two variables is 
modelled by a copula function with the generator $\phi_{1}$, i.e. $z_{1}=C\left(u_{1}, u_{2}\right)=\phi_{1}\left\{\phi_{1}^{-1}\left(u_{1}\right)+\right.$ $\left.\phi_{1}^{-1}\left(u_{2}\right)\right\}$. At the second level an another copula function is used to model the dependency between $z_{1}$ and $u_{3}$, etc. Note that the generators $\phi_{i}$ can come from the same family and they differ only through the parameter or, to introduce more flexibility, they come from different generator families. As an alternative to the fully nested model, we can consider copula functions, with arbitrary chosen combinations at each copula level. Okhrin, Okhrin and Schmid (2009a) provide several methodologies in determining the structure of the HAC from the data. The case of $d=3$ which we use further in applications is quite a simple one. If $\tau_{12}, \tau_{13}$ and $\tau_{23}$ are Kendall's $\tau$, pairwise rank correlation coefficients, we join together those $X_{i}$ and $X_{j}$ such that $\max _{i, j \in\{1,2,3\}, i \neq j}=\tau_{i j}$. Next we introduce $z=\widehat{C}\left\{\hat{F}_{i}\left(X_{i}\right), \hat{F}_{i}\left(X_{j}\right)\right\}$. Estimation techniques will be considered later. Variable $X_{i^{*}}, i^{*} \in$ $\{1,2,3\} /\{i, j\}$ is joined afterwards with the $z$.

Whelan (2004) provides tools for generating samples from Archimedean copulae, Savu and Trede (2006) derived the density of such copulae and Joe (1997) proves their positive quadrant dependence (see Theorem 4.4). Okhrin et al. (2009a) and Okhrin, Okhrin and Schmid (2009b) considered methods for determining the optimal structure of the HAC, provided asymptotic theory for the estimated parameters and derive theoretical properties of this copula family.

\section{Monte Carlo Simulation}

The Monte-Carlo simulation is often a single reliable solution to many financial problems. Within the simulation study the random variables are generated from some prescribed distributions. There are numerous methods of simulating from copula-based distributions, see Frees and Valdez (1998), Whelan (2004), Marshall and Olkin (1988),McNeil (2008), Embrechts, McNeil and Straumann (1999), Frey and McNeil (2003), Devroye (1986), etc. Here we focus on two of them, on the conditional inversion method and on the method proposed by Marshall and Olkin (1988) for Archimedean copulae with generalizations to hierarchical Archimedean copulae by McNeil (2008).

\subsection{Conditional Inverse Method}

The simulation from $d$ pseudo random variables with joint distribution defined by a copula $C$ and $d$ marginal distributions $F_{j}, j=1, \ldots, d$, may follow different techniques.

Defining the copula $j$-dimensional marginal distribution $C_{j}$ for $j=2, \ldots, d-1$ as $C_{j}\left(u_{1}, \ldots, u_{j}\right)=$ $C\left(u_{1}, \ldots, u_{j}, 1, \ldots, 1\right)$ and the derivative of $C_{j}$ with respect to the first $j-1$ arguments as

$$
c_{j-1}^{j}\left(u_{1}, \ldots, u_{j}\right)=\frac{\partial^{j-1} C_{j}\left(u_{1}, \ldots, u_{j}\right)}{\partial u_{1}, \ldots, \partial u_{j-1}}
$$

the probability $P\left(U_{j} \leq u_{j}, U_{1}=u_{1}, \ldots, U_{j-1}=u_{j-1}\right)$ can be written as

$$
\begin{aligned}
& \lim _{\substack{\Delta u_{1}, \ldots, \Delta u_{j-1} \rightarrow 0 \\
=c_{j-1}^{j}}} \frac{C_{j}\left(u_{1}+\Delta u_{1}, \ldots, u_{j-1}+\Delta u_{j-1}, u_{j}\right)-C_{j}\left(u_{1}, \ldots, u_{j}\right) .}{\Delta u_{1}, \ldots, \Delta u_{j-1}} \\
& \quad
\end{aligned}
$$


Thus, the conditional distribution $\Lambda\left(u_{j}\right)$ (given fixed $\left.u_{1}, \ldots, u_{j-1}\right)$ is a function of the ratio of derivatives:

$$
\begin{aligned}
\Lambda\left(u_{j}\right) & =P\left(U_{j} \leq u_{j} \mid U_{1}=u_{1}, \ldots, U_{j-1}=u_{j-1}\right) \\
& =\frac{c_{j-1}^{j}\left(u_{1}, \ldots, u_{j}\right)}{c_{j-1}^{j-1}\left(u_{1}, \ldots, u_{j-1}\right)}
\end{aligned}
$$

The generation of $d$ pseudo random numbers with given marginal distributions $F_{j}, j=$ $1, \ldots, d$ and dependence structure given by the copula $C$ follows the steps:

1. generate iid $v_{1}, \ldots, v_{d} \sim U[0,1]$.

2. for $j=1, \ldots, d$ calculate $u_{j}=\Lambda^{-1}\left(v_{j}\right)$.

3. set $x_{j}=F_{j}^{-1}\left(u_{j}\right)$.

\subsection{Marshal-Olkin Method}

The Marshal-Olkin method is developed for the simulations only from Archimedean copulae. The idea this approach is based on the fact that the Archimedean copulae are derived from Laplace transforms. Let $M$ be a univariate cdf of a positive random variable (so that $M(0)=0$ ) and $\phi$ be the Laplace transform of $M$, i.e.

$$
\phi(s)=\int_{0}^{\infty} \exp \{-s w\} d M(w), s \geq 0
$$

For any univariate distribution function $F$, a unique distribution $G$ exists:

$$
F(x)=\int_{0}^{\infty} G^{\alpha}(x) d M(\alpha)=\phi\{-\log G(x)\} .
$$

Considering $d$ different univariate distributions $F_{1}, \ldots, F_{d}$, we obtain

$$
C\left(u_{1}, \ldots, u_{d}\right)=\int_{0}^{\infty} \prod_{i=1}^{d} G_{i}^{\alpha} d M(\alpha)=\phi\left[\sum_{i=1}^{d} \phi^{-1}\left\{F_{i}\left(u_{i}\right)\right\}\right]
$$

which is a multivariate distribution function. By replacing the product of univariate distributions $G_{i}$ for $i=1, \ldots, d$ with an arbitrary copula function $R$ we get:

$$
C\left(u_{1}, \ldots, u_{d}\right)=\int_{0}^{\infty} \ldots \int_{0}^{\infty} R\left(G_{1}^{\alpha}, \ldots, G_{d}^{\alpha}\right) d M(\alpha) .
$$

Note that for the classical Archimedean copula $R$ is equal to a product copula.

One proceeds with the following three steps to make a draw from a distribution described by an Archimedean copula:

1. generate an observation $u$ from $M$;

2. generate an observations $\left(v_{1}, \ldots, v_{d}\right)$ from $R$; 
3. the generated vector is computed by $x_{j}=G_{j}^{-1}\left(v_{j}^{1 / u}\right)$.

This method works faster than the conditional inverse technique. The drawback is that the distribution $M$ can be determined explicitly only for a few generator functions $\phi$ like, for example for the Frank, Gumbel and Clayton families. The same problem arises in the case of hierarchical copulae, where $\phi_{i} \circ \phi_{i+1}^{-1}$ should satisfy the properties of generator functions.

\section{Copula Estimation}

The estimation of a copula based multivariate distribution involves both the estimation of the copula parameters $\theta$ and the estimation of the margins $F_{j}, j=1, \ldots, d$, however all the parameters from the copula and from the margins could be also estimated in one step. The properties and goodness of the estimator of $\theta$ heavily depend on the estimators of $F_{j}, j=1, \ldots, d$. We distinguish between a parametric and a nonparametric specification of the margins. If we are interested only in the dependency structure, the estimator of $\left\{\delta_{1}, \ldots, \delta_{d}, \theta\right\}$ should be independent of any parametric models for the margins. In practical applications, however, we are interested in a complete distribution model and, therefore, parametric models for margins are preferred.

For nonparametrically estimated margins, one may show the consistency and asymptotic normality of maximum-likelihood (ML) estimators and derive the moments of the asymptotic distribution. The ML estimation can be performed simultaneously for the parameters of the margins and of the copula function. Alternatively, a two-stage procedure can be applied, where we estimate the parameters of margins at the first stage and the copula parameters at the second stage.

Let $X$ be a $d$-dimensional random variable with parametric univariate marginal distributions $F_{j}\left(x_{j} ; \delta_{j}\right), j=1, \ldots, d$. Further let a copula belong to a parametric family $\mathcal{C}=\left\{C_{\theta}, \theta \in \Theta\right\}$. The distribution of $X$ can be expressed as

$$
F\left(x_{1}, \ldots, x_{d}\right)=C\left\{F_{1}\left(x_{1} ; \delta_{1}\right), \ldots, F_{d}\left(x_{d} ; \delta_{d}\right) ; \theta\right\}
$$

and its density as

$$
f\left(x_{1}, \ldots, x_{d} ; \delta_{1}, \ldots, \delta_{d}, \theta\right)=c\left\{F_{1}\left(x_{1} ; \delta_{1}\right), \ldots, F_{d}\left(x_{d} ; \delta_{d}\right) ; \theta\right\} \prod_{j=1}^{d} f_{j}\left(x_{j} ; \delta_{j}\right)
$$

where $c(\cdot)$ is the copula density (??). For a sample of observations $\left\{x_{t}\right\}_{t=1}^{T}, x_{t}=\left(x_{1, t}, \ldots, x_{d, t}\right)^{\top}$ and a vector of parameters $\alpha=\left(\delta_{1}, \ldots, \delta_{d}, \theta\right)^{\top} \in \mathbb{R}^{d+1}$ the likelihood function is given by

$$
L\left(\alpha ; x_{1}, \ldots, x_{T}\right)=\prod_{t=1}^{T} f\left(x_{1, t}, \ldots, x_{d, t} ; \delta_{1}, \ldots, \delta_{d}, \theta\right)
$$

and the log-likelihood function by

$$
\begin{aligned}
\ell\left(\alpha ; x_{1}, \ldots, x_{T}\right) & =\sum_{t=1}^{T} \log c\left\{F_{1}\left(x_{1, t} ; \delta_{1}\right), \ldots, F_{d}\left(x_{d, t} ; \delta_{d}\right) ; \theta\right\} \\
& +\sum_{t=1}^{T} \sum_{j=1}^{d} \log f_{j}\left(x_{j, t} ; \delta_{j}\right) .
\end{aligned}
$$


The vector of parameters $\alpha=\left(\delta_{1}, \ldots, \delta_{d}, \theta\right)^{\top}$ contains $d$ parameters $\delta_{j}$ from the marginals and the copula parameter $\theta$. All these parameters can be estimated in one step. For practical applications, however, a two step estimation procedure is more efficient.

\subsection{FML - Full Maximum Likelihood Estimation}

In the Maximum Likelihood estimation method (also called full maximum likelihood), the vector of parameters $\alpha$ is estimated in one single step through

$$
\tilde{\alpha}_{F M L}=\underset{\alpha}{\arg \max } \ell(\alpha)
$$

The estimates $\tilde{\alpha}_{F M L}=\left(\tilde{\delta}_{1}, \ldots, \tilde{\delta}_{d}, \tilde{\theta}\right)^{\top}$ solve

$$
\left(\partial \ell / \partial \delta_{1}, \ldots, \partial \ell / \partial \delta_{d}, \partial \ell / \partial \theta\right)=0 .
$$

Following the standard theory on ML estimation it is efficient and asymptotically normal. However, it is often computationally demanding to solve the system simultaneously.

\section{$5.2 \quad$ IFM - Inference for Margins}

In the IFM (inference for margins) method, the parameters $\delta_{j}$ from the marginal distributions are estimated in the first step and used to estimate the dependece parameter $\theta$ in the second step:

1. for $j=1, \ldots, d$ the log-likelihood function for each of the marginal distributions are

$$
\ell_{j}\left(\delta_{j}\right)=\sum_{t=1}^{T} \log f_{j}\left(x_{j, t} ; \delta_{j}\right)
$$

and the estimated parameters

$$
\hat{\delta}_{j}=\underset{\delta}{\arg \max } \ell_{j}\left(\delta_{j}\right)
$$

2. the pseudo log-likelihood function

$$
\ell\left(\theta, \hat{\delta}_{1}, \ldots, \hat{\delta}_{d}\right)=\sum_{t=1}^{T} \log c\left\{F_{1}\left(x_{1, t} ; \hat{\delta}_{1}\right), \ldots, F_{d}\left(x_{d, t} ; \hat{\delta}_{d}\right) ; \theta\right\}
$$

is maximised over $\theta$ to get the dependence parameter estimate $\hat{\theta}$.

The estimates $\hat{\alpha}_{I F M}=\left(\hat{\delta_{1}}, \ldots, \hat{\delta_{d}}, \hat{\theta}\right)^{\top}$ solve

$$
\left(\partial \ell_{1} / \partial \delta_{1}, \ldots, \partial \ell_{d} / \partial \delta_{d}, \partial \ell / \partial \theta\right)=0 \text {. }
$$

Detailed discussion on this method could be found in Joe and Xu (1996) Note, that this procedure does not lead to efficient estimators, however, as argued by Joe (1997) the loss in the efficiency is modest. The advantage of the inference for margins procedure lies in the dramatic reduction of the numerical complexity. Detailed discussion on the inference for margins procedure can be found in Joe and $\mathrm{Xu}$ (1996). Note, that this method does not lead to efficient estimators, however, as argued by Joe (1997) the loss in the efficiency is modest. 


\subsection{CML - Canonical Maximum Likelihood}

In the CML (canonical maximum likelihood) method, the univariate marginal distributions are estimated through the edf $\hat{F}$. The asymptotic properties of the multistage estimators of $\theta$ do not depend explicitly on the type of the nonparametric estimator, but on its convergence properties. For $j=1, \ldots, d$

$$
\hat{F}_{j}(x)=\frac{1}{T+1} \sum_{t=1}^{T} \mathbf{I}\left(x_{j, t} \leq x\right) .
$$

The pseudo log-likelihood function is

$$
\ell(\theta)=\sum_{t=1}^{T} \log c\left\{\hat{F}_{1}\left(x_{1, t}\right), \ldots, \hat{F}_{d}\left(x_{d, t}\right) ; \theta\right\}
$$

and the copula parameter estimator $\hat{\theta}_{C M L}$ is given by

$$
\hat{\theta}_{C M L}=\underset{\theta}{\arg \max } \ell(\theta) \text {. }
$$

Notice that the first step of the IMF and CML methods estimates the marginal distributions. After marginals are estimated, a pseudo sample $\left\{u_{t}\right\}$ of observations transformed in the unit $d$-cube is obtained and used in the copula estimation. As in the IFM, the semiparametric estimator $\hat{\theta}$ is asymptotically normal under suitable regularity conditions.

\section{Asset Allocation}

We illustrate the extension of the classical asset allocation problem to copula-based models. We consider an investor with a CRRA utility function $U(x)=(1-\gamma)^{-1} x^{1-\gamma}$ willing to allocate his wealth to $d$ risky assets. We denote the $d$-dimensional vector of $d$ asset prices by $S_{t}=\left(S_{1, t}, \ldots, S_{d, t}\right)^{\top}$ and their continuously compounded asset returns at time $t+1$ by $X_{t+1}=\left(X_{1, t+1}, \ldots, X_{d, t+1}\right)^{\top}$ where $X_{t+1}=\log S_{t+1}-\log S_{t}$. The vector of portfolio weights by $w=\left(w_{1}, \ldots, w_{d}\right)^{\top}$. Let $F_{t+1}$ be the $d$-dimensional distribution function of $X_{t+1}$ with the mean $\mu_{t+1}$ and covariance matrix $\Sigma_{t+1}$. The aim is to forecast $F_{t+1}$ for the time period $t+1$ using the data up to time $t$. The estimator is denoted by $\hat{F}_{t+1}$ with the mean $\hat{\mu}_{t+1}$, the covariance matrix $\hat{\Sigma}_{t+1}$ and the density $\hat{f}_{t+1}$. The objective of the investor is to maximise the expected utility at the time point $t+1$. This leads to the optimisation problem

$$
\max _{w \in \mathcal{W}} \mathrm{E}_{\hat{F}_{t+1}} U\left(1+w^{\top} X_{t+1}\right) .
$$

In the case of no short sales constraint we set $\mathcal{W}=\left\{w \in[0,1]^{d}: w^{\top} 1=1\right\}$ else we set $\mathcal{W}=\left\{w \in \mathbb{R}^{d}: w^{\top} 1=1\right\}$. The conditional expectation in (5) implies that we integrate the utility with respect to the forecasted distribution $\hat{F}_{t+1}$. This reduces the problem (5) to the problem

$$
\max _{w \in \mathcal{W}} \int \cdots \int U\left(1+w^{\top} X_{t+1}\right) \hat{f}_{t+1}\left(X_{t+1}\right) d X_{t+1} .
$$


There are several alternative parametric approaches to modelling $F_{t+1}$. Let $\Sigma_{d, t+1}$ denote the diagonal matrix containing only the main diagonal of $\Sigma_{t+1}$. Then $\Sigma_{t+1}=$ $\Sigma_{d, t+1}^{1 / 2} R_{t+1} \Sigma_{d, t+1}^{1 / 2}$, where $R_{t+1}$ denotes the correlation matrix. A standard approach is to define the model of the asset returns in the form

$$
\Sigma_{d, t}^{-1 / 2}\left(X_{t}-\mu_{t}\right) \sim \mathrm{N}_{d}\left(0, R_{t}\right),
$$

where the conditional moments $\mu_{t}$ and $\Sigma_{t}$ are modelled by a GARCH type process.

To introduce a copula-based distribution into the asset allocation we deviate from the normality assumption and assume that $F=C\left(F_{1}, \ldots, F_{d}\right)$. Thus $(7)$ is replaced by:

$$
\Sigma_{d, t}^{-1 / 2}\left(X_{t}-\mu_{t}\right) \sim C\left(F_{1}, \ldots, F_{d}\right)
$$

with some given functional forms of the copula and the marginal distributions. Similarly as above, the parameters of the conditional moments of the copula and of the marginal distributions are estimated using the ML method.

In Patton (2004) the investor allocates his wealth between small cap and large cap stocks (i.e. $d=2$ ). The conditional mean is defined as linear function of the lagged asset returns and additional explanatory variables. The conditional variance is stated in the $\operatorname{TARCH}(1,1)$ form. The rotated Gumbel copula with skewed $t$ margins are used to construct the bivariate distribution of the residuals. This model reveals the highest likelihood function and the lowest AIC and BIC criterion. It is concluded that unconstrained portfolios derived from the normality assumption performed worse in 9 of 10 different trading strategies compared to the Gumbel model.

\section{Value-at-Risk of the Portfolio Returns}

If the return of the stock $i$ at time point $t$ is denoted as $X_{i t}$ then the portfolio value $V$ at time $t$ is defined recursively as

$$
V_{t}=V_{t-1}\left(1+\sum_{i=1}^{d} w_{i} X_{i t}\right),
$$

where $w_{i}$ for $i=1, \ldots, d$ are the corresponding portfolio weights. Ruled with this notation the portfolio return is then given by

$$
R_{t p}=\frac{V_{t}}{V_{t-1}}-1=\sum_{i=1}^{d} X_{i t} w_{i}
$$

In our study we consider the case of equally weighted portfolio, i.e. $w_{i}=\frac{1}{d}$ for $i=1, \ldots, d$. The portfolio return is the random variable and its distribution strongly depends on the underlying distribution of the indices.

The distribution function of $R_{p}$, dropping the time index, is given by

$$
F_{R_{p}}(\xi)=\mathrm{P}\left(R_{p} \leq \xi\right) .
$$

One of the main advantages of copulae is the fact that they allow flexible modelling of the tail behaviour of multivariate distributions. Since the tail behaviour explains the 
simultaneous outliers of asset returns, it is of special interest in risk management. The Value-at-Risk of a portfolio at level $\alpha$ is defined as the lower $\alpha$-quantile of the distribution of the portfolio return, i.e.

$$
\operatorname{VaR}(\alpha)=F_{R_{p}}^{-1}(\alpha)
$$

The VaR is a reasonable measure of risk if we assume that the returns are elliptically distributed. Moreover, the assumption of ellipticity implies that minimising the variance in the Markowitz problem also minimises the $\mathrm{VaR}$, the expected shortfall and any other coherent measure of risk. However, this statement is false in the non-elliptical case. Moreover, regarding the effect of diversification the variance is the smallest (highest) for perfect negative (positive) correlation of the assets. This also holds for the VaR in the elliptical case, however, not for the non-elliptical distributions. This implies that for copula based distribution the VaR should be used with caution and its computation should be awarded more attention. Detailed description of the VaR estimation procedure at prescribed level $\alpha$ can be found in Giacomini and Härdle (2005).

Our aim is to determine such $\xi$ that $\mathrm{P}\left(R_{p} \leq \xi\right)=\alpha$. Note that

$$
R_{p}=w^{\top} X=\sum_{i=1}^{d} w_{i} X_{i}=\sum_{i=1}^{d} w_{i} F_{i}^{-1}\left(u_{i}\right)
$$

where $F_{i}$ denotes the marginal distributions of individual asset returns, $u_{i}=F_{i}\left(X_{i}\right) \sim$ $U[0,1]$ for all $i=1, \ldots, d$ and $u_{1}, \ldots, u_{d} \sim C$. The copula $C$ defines the dependency structure between the asset returns. This implies that

$$
F_{R_{p}}(\xi)=\mathrm{P}\left(R_{p} \leq \xi\right)=\int_{\mathcal{U}} c\left(u_{1}, \ldots, u_{d}\right) d u_{1} \ldots d u_{d}
$$

with

$$
\mathcal{U}=\left\{[0,1]^{d-1} \times\left[0, u_{d}(\xi)\right]\right\}, \quad u_{d}(\xi)=F_{d}\left\{\xi / w_{d}-\sum_{i=1}^{d-1} w_{i} F_{i}^{-1}\left(u_{i}\right) / w_{d}\right\} .
$$

For fixed $\alpha$, the VaR is determined by solving $(10)$ numerically for $\xi$. Direct multidimensional numerical integration is a tedious task which can be substantially simplified by using the Monte-Carlo integration. For this purpose we have to generate random samples from $C$ using the methods described in Section 4.

In the empirical study we consider four countries Canada, Germany, U.S. and U.K. from the MCSI index and eleven models of the joint multivariate distribution of indices, which include $t$-copula, Gaussian copula, simple exchangeable Archimedean copula, binary HAC and aggregated binary HAC, with normally and $t$-distributed margins. As a benchmark we use the empirical VaR, based purely on the real data.

In the cases where margins are $t$-distributed, we consider $t$-distribution with three degrees of freedom, while estimated $t$-distributions for this data are $t_{3.163}, t_{3.420}, t_{3.023}, t_{2.879}$. Multivariate $t$-copula in this case has eight degrees of freedom. Let us consider the simulation procedure, where on the first stage we estimate the covariance matrix $\widehat{\Sigma}=$ $\left\{\widehat{\Sigma}_{i j}\right\}_{i, j=1, \ldots, d}$, mean vector $\widehat{\mu}=\left\{\widehat{\mu}_{i}\right\}_{i=1, \ldots, d}$ from the real data set and assume, or estimate, the marginal distributions $\widehat{F}_{i}(\cdot)$ (in our case they are normally or $t$-distributed), for $i=$ 
$1, \ldots, d$. Next we show how to sample $u_{1}, \ldots, u_{d} \in \mathcal{U}$ from (11). First we simulate the vector $u$ of a dimension $d-1$

$$
u_{1}, \ldots, u_{d-1} \sim U(0,1) .
$$

Based on $u$ we consider $x=\left\{x_{i}\right\}_{i=1, \ldots, d-1}$ which for normal margins is equal to

$$
x_{i}=\Phi^{-1}\left(u_{i}\right) \sqrt{\widehat{\Sigma}_{i i}}+\widehat{\mu}_{i}, \quad i=1, \ldots, d-1,
$$

and for $t$ margins is

$$
x_{i}=t^{-1}\left(u_{i}\right) \sqrt{\frac{\nu_{i}-2}{\nu_{i}} \widehat{\Sigma}_{i i}}+\widehat{\mu}_{i}, \quad i=1, \ldots, d-1,
$$

where $\nu_{i}, i=1, \ldots, d$ are degrees of freedom for marginal distributions. This transformation returns a normally or $t$-distributed vector $x$ with the same parameters as the real data set.

Theoretically, in further steps we have to find bounds for the last stock (or index) to gain the portfolio $\xi$ which is the $\alpha$ quantile. Thus, we separate our maximally reachable portfolio return $\xi$ into two parts

$$
\xi=\sum_{i=1}^{d-1} \frac{1}{d} X_{i}+\frac{1}{d} X_{d}
$$

then the return of the last index given the return of the portfolio is

$$
X_{d}=d \xi-\sum_{i=1}^{d-1} X_{i}
$$

where the upper bound for our last value in vector $u$ is then

$$
u_{d}^{*}=\widehat{F}_{d}\left(d \xi-\sum_{i=1}^{d-1} x_{i}\right) .
$$

Value $u_{d}^{*}$ is uniformly distributed on $[0,1]$ and we simulate the last element of the vector $u_{d} \sim U\left(0, u_{d}^{*}\right)$.

As mentioned above, the goal is to compute 10 which for this setting is

$$
F_{R_{p}}(\xi)=\int_{[0,1]^{d-1} \times\left[0, u_{d}^{*}\right]} c\left(u_{1}, \ldots, u_{d}\right) d u_{1} \ldots d u_{d} .
$$

Then by solving $F_{R_{p}}(\xi)=\alpha$ we find $R_{\alpha}=\operatorname{VaR}(\alpha)$. In our study we solve the equations numerically using the golden section method. The integration is performed using the Monte-Carlo technique

$$
P\left(\widehat{R_{p} \leq} \xi\right)=\frac{1}{n_{s}} \sum_{i=1}^{n_{s}} c\left(u_{1 i}, \ldots, u_{d i}\right)
$$

where $n_{s}$ is equal to $10^{8}, \alpha$ is set to be $1 \%$ and the values $u_{1 i}, \ldots, u_{d i}$ for $i=1, \ldots, n_{s}$ are simulated using the method described above. The precision of $R$ is set at 0.00015 . 
Table 1: VaR for the 4-dimensional data set

\begin{tabular}{l|r|r}
\hline \hline & $\mathrm{N}$ & $t_{3}$ \\
\hline $\mathrm{N}$ & -0.0194 & -0.0210 \\
$t_{8}$ & $\mathbf{- 0 . 0 1 9 9}$ & $\mathbf{- 0 . 0 2 1 3}$ \\
$A C$ & -0.0174 & -0.0154 \\
$H A C_{\text {binary }}$ & -0.0187 & -0.0194 \\
$H A C_{\text {binary aggr. }}$ & -0.0188 & -0.0194 \\
\hline Empirical & \multicolumn{2}{|c}{-0.0235} \\
\hline \hline
\end{tabular}

The final results for all methods are given in Table 1. In the left-hand column we provide the models with normal margins and in the right-hand column with $t$ margins. From top to bottom we have five different copula functions like Gaussian, $t$, simple Archimedean copula, binary HAC and binary aggregated HAC. The empirical VaR which is at the bottom of the table is derived from the empirical quantile. Bold fonts in the table emphasize those results which are closest in absolute value to the empirical one in each column, and italic fonts the worst cases in absolute value.

As can be seen from Table 1, the results which are the best in absolute value are those returned by the model with $t$-copula and $t$ margins. The model based on the simple Archimedean copula is the worst one. This is quite natural, since this copula needs exchangeability between variables, which is not observable here (see previous section). HAC with binary as well as aggregated binary structures, unfortunately, give us results that are not much worse compared to $t$-copula and Gaussian copula. For VaR(0.01) the $t$-copula with $t$ margins provided the best result.

\subsection{VaR of the P\&L}

This sub-section introduces the main assumptions and steps necessary to estimate the VaR from a Profit and Loss of a linear portfolio using copulae. Static and time-varying methods and their VaR performance evaluation through backtesting are described below.

In this section $w$ is the portfolio, which is represented by the number of assets for a specified stock in the portfolio, $w=\left\{w_{1}, \ldots, w_{d}\right\}, w_{i} \in \mathbb{Z}$. The value $V_{t}$ of the portfolio $w$ is given non-recursively by

$$
V_{t}=\sum_{j=1}^{d} w_{j} S_{j, t}
$$

and the random variable

$$
\begin{aligned}
L_{t+1} & =\left(V_{t+1}-V_{t}\right) \\
& =\sum_{j=1}^{d} w_{j} S_{j, t}\left\{\exp \left(X_{j, t+1}\right)-1\right\} .
\end{aligned}
$$

also called profit and loss (PESL) function, expresses the absolute change in the portfolio value in one period. 
Similarly to the previous case, the distribution function of $L$, dropping the time index, is given by

$$
F_{L}(x)=\mathrm{P}(L \leq x)
$$

As usual the Value-at-Risk at level $\alpha$ from a portfolio $w$ is defined as the $\alpha$-quantile from $F_{L}$ :

$$
\operatorname{VaR}(\alpha)=F_{L}^{-1}(\alpha)
$$

It follows from $(13)$ that $F_{L}$ depends on the $d$-dimensional distribution of log-returns $F_{X}$. In general, the loss distribution $F_{L}$ depends on a random process representing the risk factors influencing the $\mathrm{P} \& \mathrm{~L}$ from a portfolio. In the present case log-returns are a suitable risk factor choice. Thus, modelling their distribution is essential to obtain the quantiles from $F_{L}$.

Contrary to the previous section, here log-returns are assumed to be time-dependent, thus a log-returns process $\left\{X_{t}\right\}$ can be modelled as

$$
X_{j, t}=\mu_{j, t}+\sigma_{j, t} \varepsilon_{j, t}
$$

where $\varepsilon_{t}=\left(\varepsilon_{1, t}, \ldots, \varepsilon_{d, t}\right)^{\top}$ are standardised $i . i . d$. innovations with $\mathrm{E}\left[\varepsilon_{j, t}\right]=0$ and $\mathrm{E}\left[\varepsilon_{j, t}^{2}\right]=$ 1 for $j=1, \ldots, d ; \mathcal{F}_{t}$ is the available information at time $t$ :

$$
\mu_{j, t}=E\left[X_{j, t} \mid \mathcal{F}_{t-1}\right]
$$

is the conditional mean given $\mathcal{F}_{t-1}$ and

$$
\sigma_{j, t}^{2}=E\left[\left(X_{j, t}-\mu_{j, t}\right)^{2} \mid \mathcal{F}_{t-1}\right]
$$

is the conditional variance given $\mathcal{F}_{t-1}$. The innovations $\varepsilon=\left(\varepsilon_{1}, \ldots, \varepsilon_{d}\right)^{\top}$ have joint distribution

$$
F_{\varepsilon}\left(\varepsilon_{1}, \ldots, \varepsilon_{d}\right)=C_{\theta}\left\{F_{1}\left(\varepsilon_{1}\right), \ldots, F_{d}\left(\varepsilon_{d}\right)\right\}
$$

where $C_{\theta}$ is a copula belonging to a parametric family $\mathcal{C}=\left\{C_{\theta}, \theta \in \Theta\right\}$, and $F_{j}, j=$ $1, \ldots, d$ are continuous marginal distributions of $\varepsilon_{j}$. To obtain the Value-at-Risk in this set up, the dependence parameter and distribution function from residuals are estimated from a sample of log-returns and used to generate P\&L Monte Carlo samples. Their quantiles at different levels are the estimators for the Value-at-Risk.

For a portfolio $w$ on $d$ assets and a sample $\left\{x_{j, t}\right\}_{t=1}^{T}, j=1, \ldots, d$ of log-returns, the Value-at-Risk at level $\alpha$ is estimated according to the following steps:

1. Estimation of residuals $\hat{\varepsilon}_{t}$ from the prespecified time-series model;

2. Specification and estimation of marginal distributions $F_{j}\left(\hat{\varepsilon}_{j}\right)$;

3. Specification of a parametric copula family $\mathcal{C}$ and estimation of dependence parameter $\theta$;

4. Generation of Monte Carlo sample of innovations $\varepsilon$ and losses $L$, for the forecast on the one day; 
5. Estimation of $\widehat{\operatorname{VaR}}(\alpha)$, the empirical $\alpha$-quantile from the forecasted $L$.

The application of the (static) procedure described above on sliding windows of a time series $\left\{x_{j, t}\right\}_{t=1}^{T}$ delivers a sequence of parameters for a copula family. Hence the denomination time-varying copulae.

Using moving windows of size $r$ in time $t$

$$
\left\{x_{t}\right\}_{t=s-w+1}^{s}
$$

for $s=r, \ldots, T$, the procedure described in the section above generates the time series $\left\{\widehat{V a R}_{t}\right\}_{t=r}^{T}$ of Value-at-Risk and $\left\{\hat{\theta}_{t}\right\}_{t=r}^{T}$ dependence parameters estimates.

Afterwards Backtesting is used to evaluate the performance of the specified copula family $\mathcal{C}$. The estimated values for the VaR are compared with the true realisations $\left\{l_{t}\right\}$ of the $\mathrm{P} \& \mathrm{~L}$ function, an exceedance occuring for each $l_{t}$ smaller than $\widehat{V a R}_{t}(\alpha)$. The ratio of the number of exceedances to the number of observations gives the exceedances ratio $\hat{\alpha}$ :

$$
\hat{\alpha}=\frac{1}{T-r} \sum_{t=r}^{T} \mathbf{I}\left\{l_{t}<\widehat{\operatorname{VaR}}_{t}(\alpha)\right\} .
$$

The estimation methods described before are used on two portfolio, the first composed of 2 positions, the second of 3 positions. Different copulae are used in static and dynamic setups and their VaR performance is compared based on backtesting.

In this section, the Value-at-Risk of portfolios for two companies (Tyssenkrupp (TKA) and Volkswagen (VOW) from 01.12.1997 to 03.07.2007) is computed using different copulae.

Assuming the log-returns $\left\{X_{j, t}\right\}$ follow a $\operatorname{GARCH}(1,1)$ process we have

$$
X_{j, t}=\mu_{j, t}+\sigma_{j, t} \varepsilon_{j, t}
$$

where

$$
\sigma_{j, t}^{2}=\omega_{j}+\alpha_{j} \sigma_{j, t-1}^{2}+\beta_{j}\left(X_{j, t-1}-\mu_{j, t-1}\right)^{2}
$$

and $\omega>0, \alpha_{j} \geq 0, \beta_{j} \geq 0, \alpha_{j}+\beta_{j}<1$.

The fit of a $\operatorname{GARCH}(1,1)$ model to the sample of log returns $\left\{x_{t}\right\}_{t=1}^{T}, X_{t}=\left(X_{1, t}, X_{2, t}\right)^{\top}$, $T=2500$, gives the estimates $\hat{\omega}_{j}, \hat{\alpha}_{j}$ and $\hat{\beta}_{j}$, as in Table 2 , and empirical residuals $\left\{\hat{\varepsilon}_{t}\right\}_{t=1}^{T}$, where $\hat{\varepsilon}_{t}=\left(\hat{\varepsilon}_{1, t}, \hat{\varepsilon}_{2, t}\right)^{\top}$. The marginal distributions are specified as normal, i.e., $\hat{\varepsilon}_{j} \sim \mathrm{N}\left(\hat{\mu}_{j}, \hat{\sigma}_{j}\right)$ with parameters $\hat{\delta}_{j}=\left(\hat{\mu}_{j}, \hat{\sigma}_{j}\right)$ estimated from the data.

Figure 1 displays the Kernel density estimator of the residuals and of the normal density, estimated with an Quartic kernel. The dependence parameters are estimated for different copula families (Gaussian, Clayton and Gumbel). Residuals $\hat{\varepsilon}$ and fitted copulae (Gaussian, Clayton and Gumbel) are plotted in Figure 2.

In the dynamic approach, the empirical residuals are sampled in moving windows with a fixed size $r=250,\left\{\hat{\varepsilon}_{t}\right\}_{t=s-r+1}^{s}$, for $s=r, \ldots, T$. The time series from estimated dependence parameters for each copula family are in Figure 3.

The same portfolio compositions as in the static case are used to generate P\&L samples. The series of estimated Value-at-Risk and the P\&L function for selected portfolios are plotted in Figure 4,5 and 6. 


\begin{tabular}{r|rrrrrr}
\hline \hline & $\hat{\mu}_{j}$ & $\hat{\omega}_{j}$ & $\hat{\alpha}_{j}$ & $\hat{\beta}_{j}$ & BL & KS \\
\hline \multirow{2}{*}{ MRK } & $7.392 \mathrm{e}-04$ & $4.588 \mathrm{e}-06$ & $3.333 \mathrm{e}-02$ & $9.572 \mathrm{e}-01$ & 0.1285 & $1.255 \mathrm{e}-11$ \\
& $(3.672 \mathrm{e}-04)$ & $(1.557 \mathrm{e}-06)$ & $(6.225 \mathrm{e}-03)$ & $(8.568 \mathrm{e}-03)$ & & \\
TKA & $7.845 \mathrm{e}-04$ & $3.549 \mathrm{e}-06$ & $7.087 \mathrm{e}-02$ & $9.252 \mathrm{e}-01$ & 0.1360 & $4.189 \mathrm{e}-05$ \\
& $(3.308 \mathrm{e}-04)$ & $(1.149 \mathrm{e}-06)$ & $(9.837 \mathrm{e}-03)$ & $(9.915 \mathrm{e}-03)$ & & \\
VOW & $9.720 \mathrm{e}-04$ & $1.239 \mathrm{e}-05$ & $9.303 \mathrm{e}-02$ & $8.830 \mathrm{e}-01$ & $1.927 \mathrm{e}-05$ & $3.422 \mathrm{e}-06$ \\
& $(3.480 \mathrm{e}-04)$ & $(2.699 \mathrm{e}-06)$ & $(1.301 \mathrm{e}-02)$ & $(1.566 \mathrm{e}-02)$ & & \\
\hline \hline
\end{tabular}

Table 2: Fitting of univariate $\operatorname{GARCH}(1,1)$ to asset returns. The standard deviation of the parameters are given in parentheses. The last two columns provide the $p$-values of the Box-Ljung test (BL) for autocorrelations and Kolmogorov-Smirnov test (KS) for normality applied to the residuals
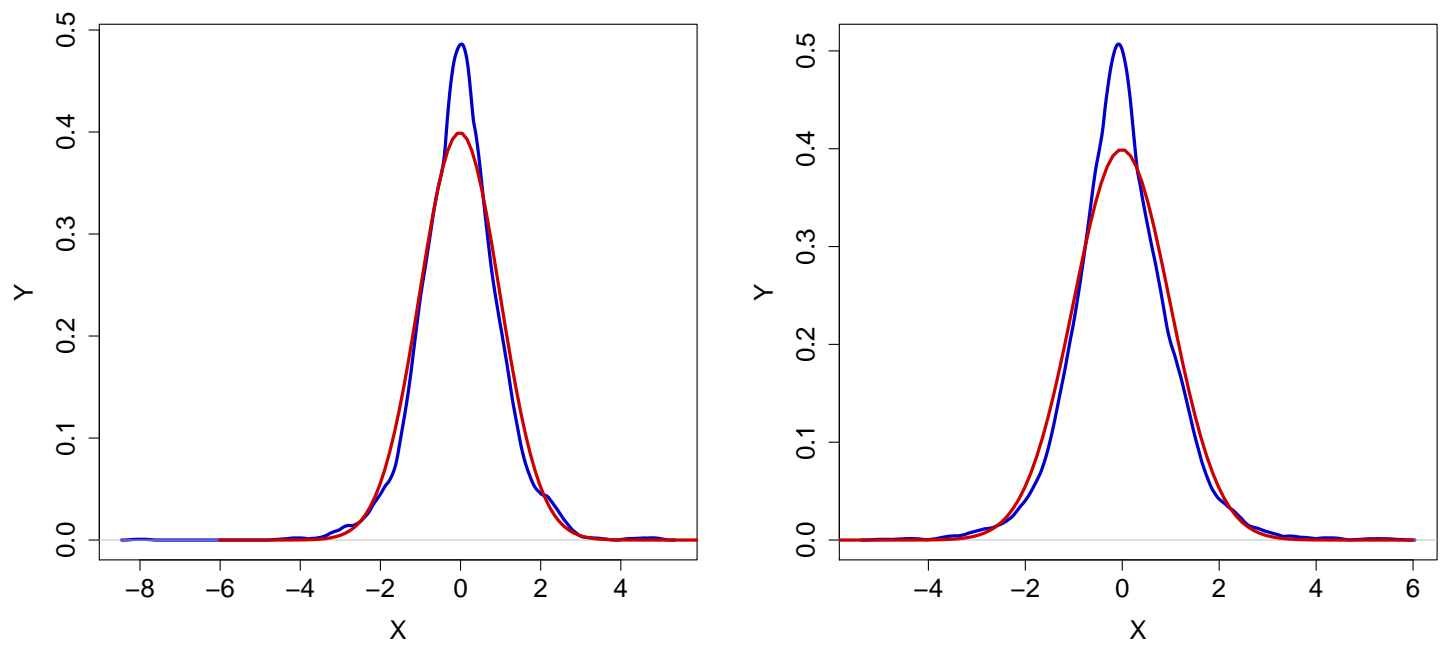

Fig. 1: Kernel density estimator of the residuals and of the normal density from TKA (left) and VOW (right). Quartic kernel, $\hat{h}=2.78 \hat{\sigma} n^{-0.2}$.
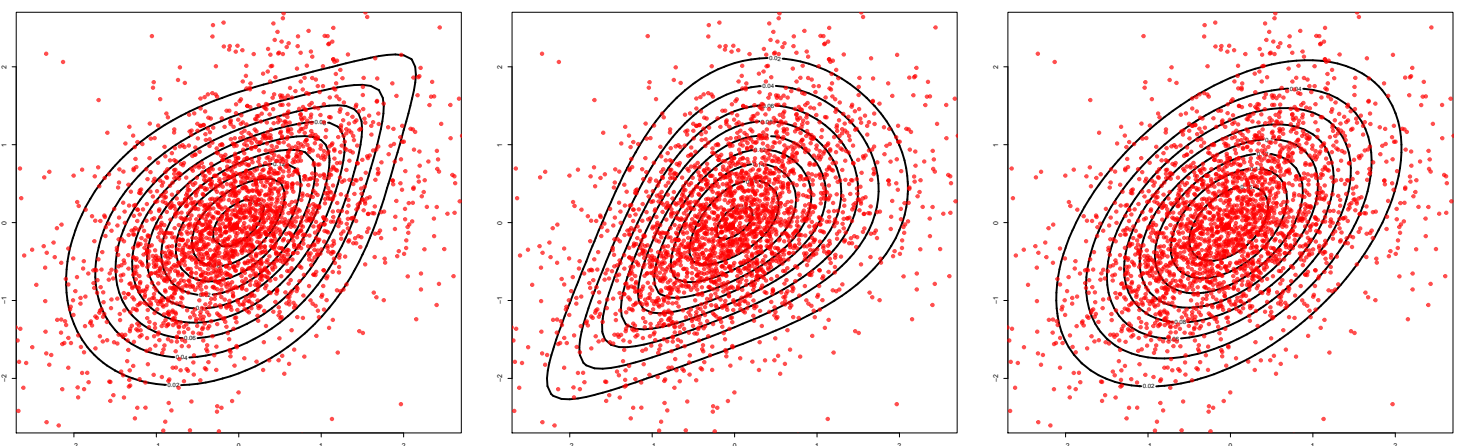

Fig. 2: Residuals $\hat{\varepsilon}$ and fitted copulae: Gaussian $(\hat{\rho}=0.462)$, Clayton $(\hat{\theta}=0.880)$, Gumbel $(\hat{\theta}=1.439)$. 

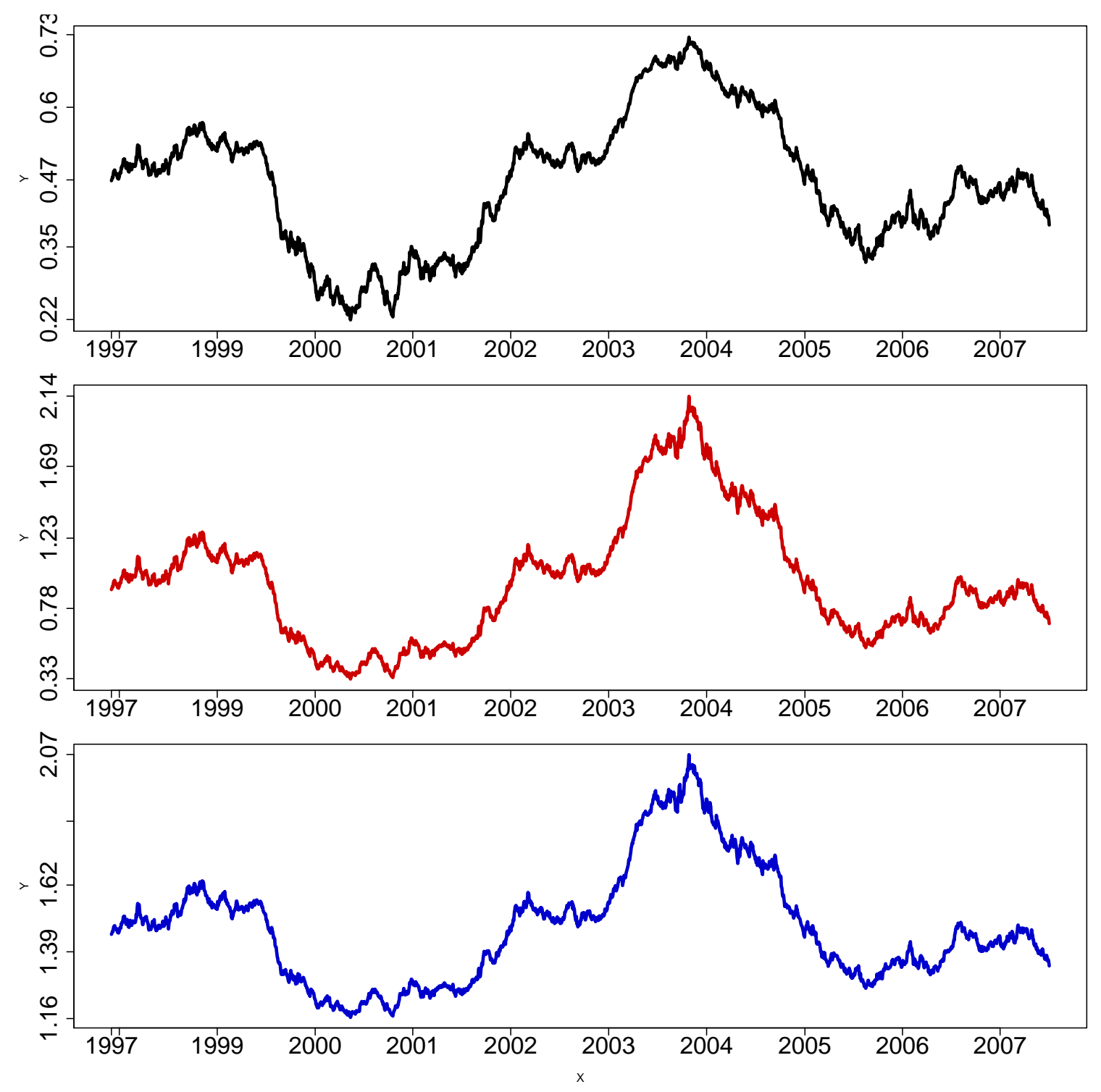

Fig. 3: Dependence parameter $\hat{\theta}$, estimated using the IFM method, Gaussian (upper panel), Gumbel (middle panel) and Clayton (lower panel) copulae, moving window $(w=$ $250)$. 


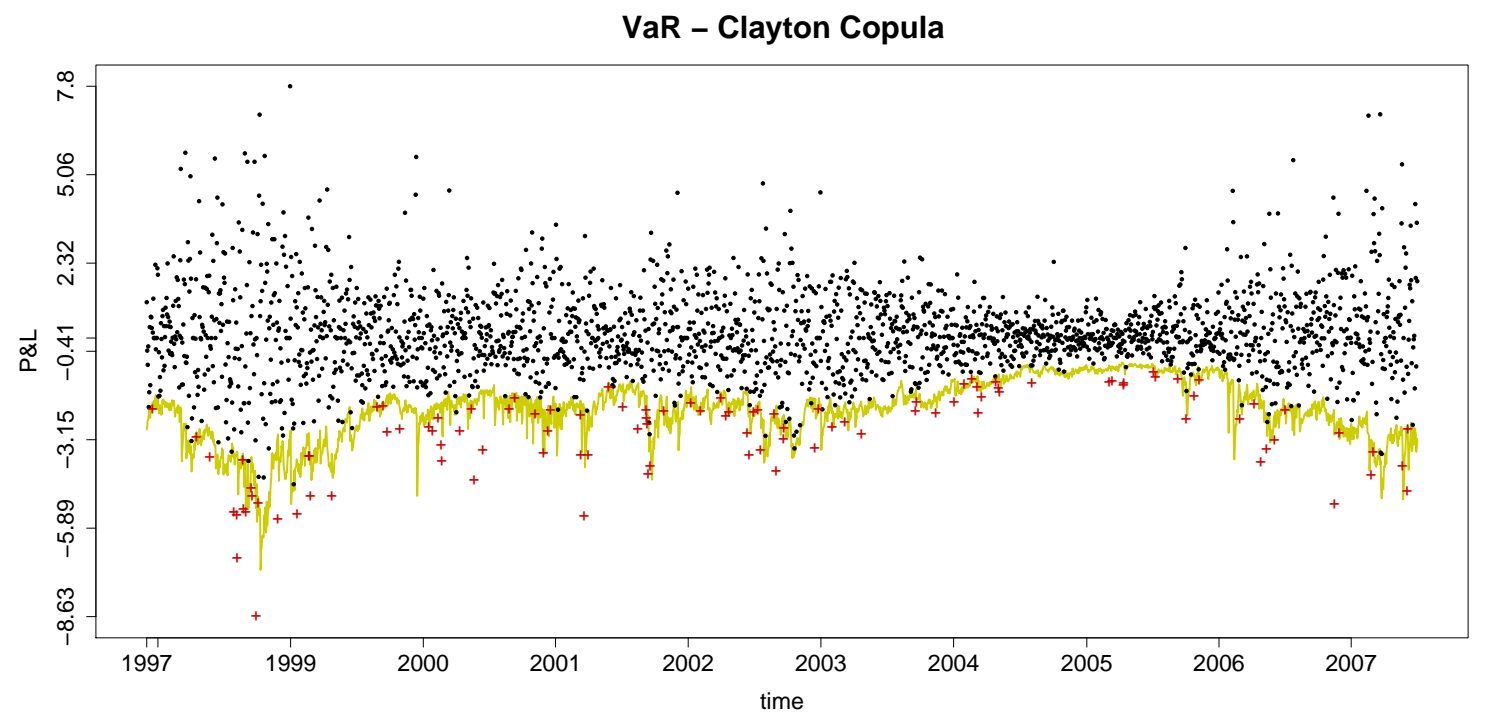

Fig. 4: $\widehat{\operatorname{VaR}}(\alpha)$ (solid line), P\&L (dots) and exceedances (crosses), $\alpha=0.05, \hat{\alpha}=0.0424$. P\&L samples generated with Clayton copula.

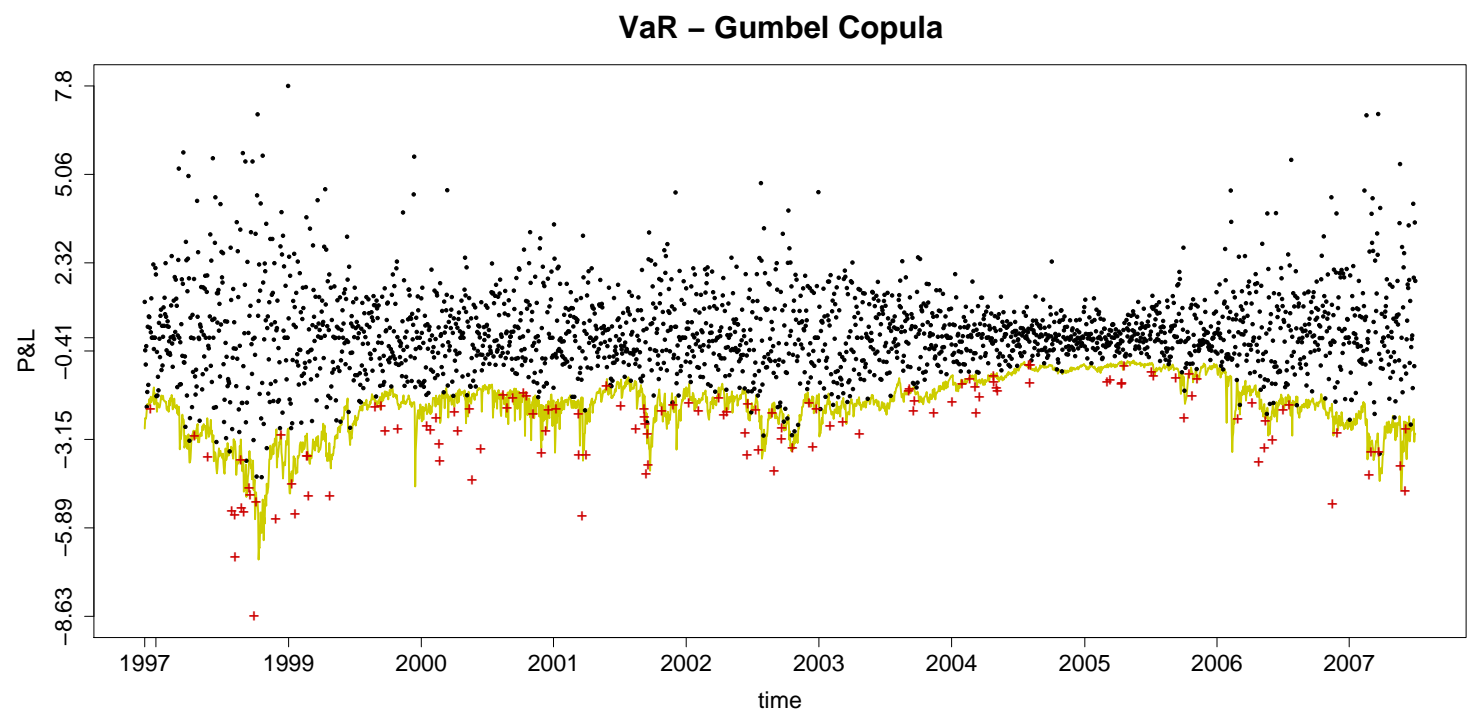

Fig. 5: $\widehat{\operatorname{VaR}}(\alpha)$ (solid line), P\&L (dots) and exceedances (crosses), $\alpha=0.05, \hat{\alpha}=0.0508$. P\&L samples generated with Gumbel copula. 


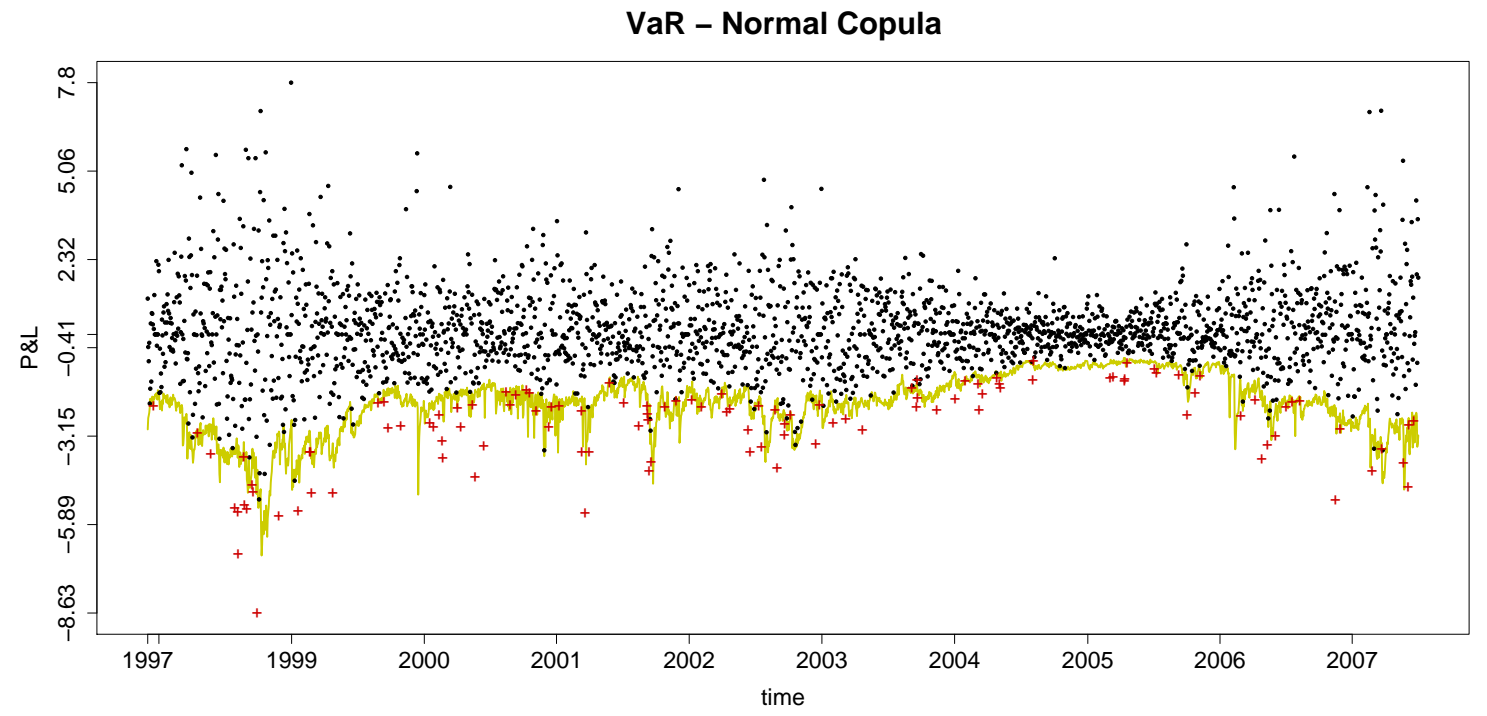

Fig. 6: $\widehat{\operatorname{VaR}}(\alpha)$ (solid line), P\&L (dots) and exceedances (crosses), $\alpha=0.05, \hat{\alpha}=0.0464$. P\&L samples generated with Gaussian copula.

\subsection{3-dimensional Portfolio}

In this section, the Value-at-Risk of portfolios composed of 3 positions (Merck (MRK), Tyssenkrupp (TKA) and Volkswagen (VOW) from 01.12.1997 to 03.07.2007) is computed using a time-varying simple Gumbel copula and time-varying hierarchical Archimedean copula with generators from the Gumbel family.

The estimation of the parameters of the 3-dimensional copula was done by the IFM method. Concerning the HAC, we determine the structure under each window and reestimate the parameters.

The fit of a GARCH(1,1) model to the sample of log returns $\left\{X_{t}\right\}_{t=1}^{T}, X_{t}=\left(X_{1, t}, X_{2, t}, X_{3, t}\right)^{\top}$, $T=2500$, gives the estimates $\hat{\omega}_{j}, \hat{\alpha}_{j}$ and $\hat{\beta}_{j}$, as in Table 2 , and empirical residuals $\left\{\hat{\varepsilon}_{t}\right\}_{t=1}^{T}$, where $\hat{\varepsilon}_{t}=\left(\hat{\varepsilon}_{1, t}, \hat{\varepsilon}_{2, t}, \hat{\varepsilon}_{3, t}\right)^{\top}$, as in upper right part of Figure 8 . The marginal distributions are specified as normal, $\hat{\varepsilon}_{j} \sim \mathrm{N}\left(\hat{\mu}_{j}, \hat{\sigma}_{j}\right)$ with the estimated parameters $\hat{\delta}_{j}=\left(\hat{\mu}_{j}, \hat{\sigma}_{j}\right)$.

The estimated Value-at-Risk at level $\alpha$ together with the P\&L function are plotted in Figure 9 for the simple Archimedean Copula (AC) and on 10 for the HAC. As can be seen from the backtesting results for different VaR levels, HAC outperforms the simple AC in all levels. This implies the necessity of dependence flexibility in modelling of log-returns.

\section{Summary}

To conclude, a summary of the main findings of this paper. We calculated the Valueat-Risk for the static and dynamic portfolio constructed by different methods. Three different copulae - Gumbel, Clayton and Gaussian - were used to estimate the Value-atRisk from the two- (MRK and TKA) and three- (MRK, TKA and VOW) dimensional portfolios. From the time series of estimated dependence parameters, we can verify that the dependence structure is represented in a similar form with all copula families, as in Figure 3 . 

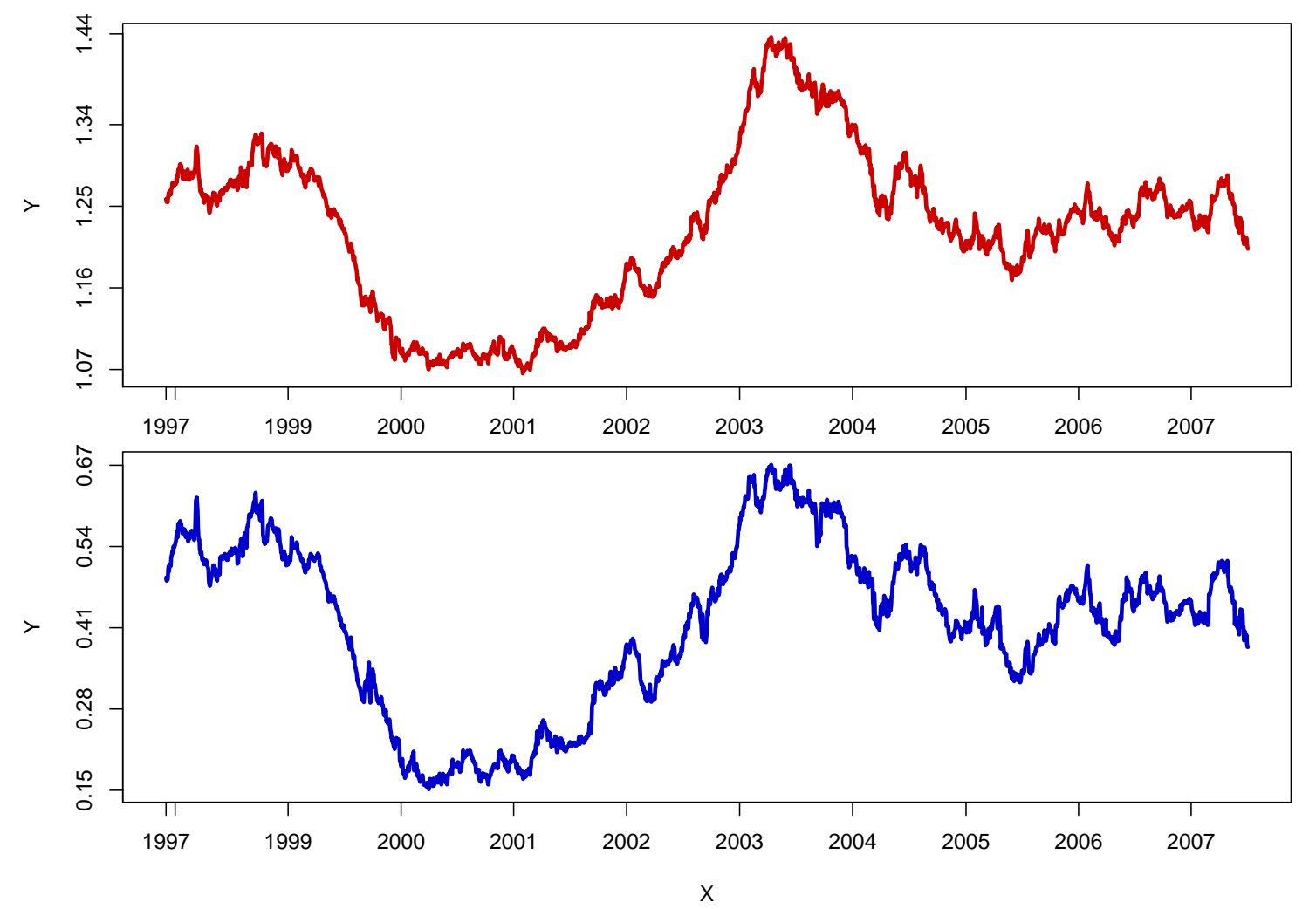

Fig. 7: Dependence parameter $\hat{\theta}$, estimated using the IFM method, Clayton (upper panel) and Gumbel (lower panel) copulae, moving window $(w=250)$.

Using backtesting results to compare the performance in the VaR estimation, we remark that on average the Clayton and Gaussian copulae overestimate the VaR. In terms of capital requirement, a financial institution computing VaR with those copulae would be requested to keep more capital aside than necessary to guarantee the desired confidence level.

The estimation with Gumbel copula, on another side, produced results close to the desired level. Gumbel copulae seems to represent specific data dependence structures (like lower tail dependencies, relevant to explain simultaneous losses) better than Gaussian and Clayton copulae.

\section{References}

Clayton, D. G. (1978). A model for association in bivariate life tables and its application in epidemiological studies of familial tendency in chronic disease incidence, Biometrika 65: $141-151$.

Deutsch, H. and Eller, R. (1999). Derivatives and Internal Models, Macmillan Press.

Devroye, L. (1986). Non-uniform Random Variate Generation, Springer Verlag, New York.

Embrechts, P., McNeil, A. J. and Straumann, D. (1999). Correlation and dependence in risk management: Properties and pitfalls, RISK pp. 69-71. 

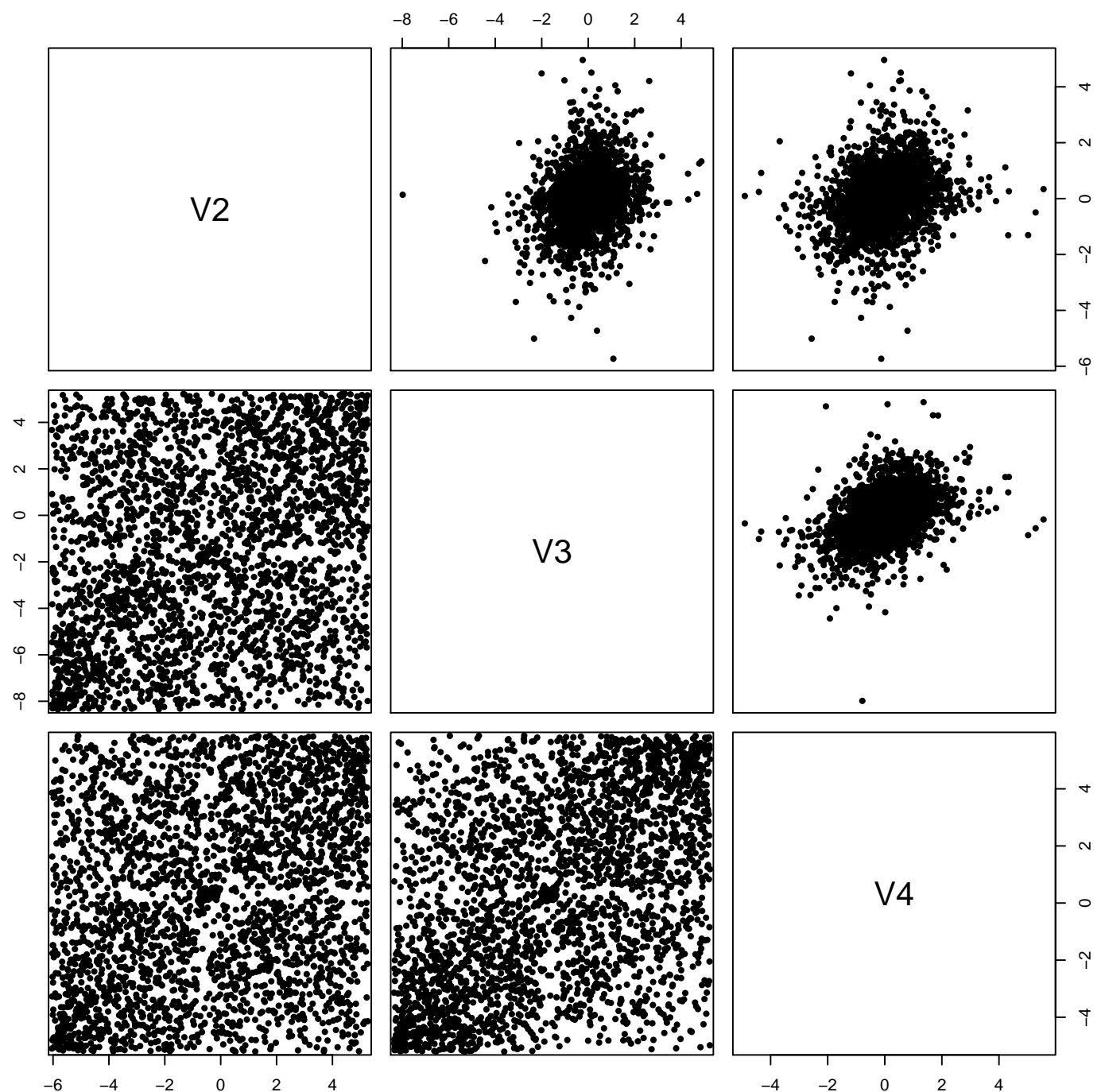

Fig. 8: Scatterplots from GARCH residulas (upper triangular) and from residuals mapped on unit square by the cdf (lower triangular). 


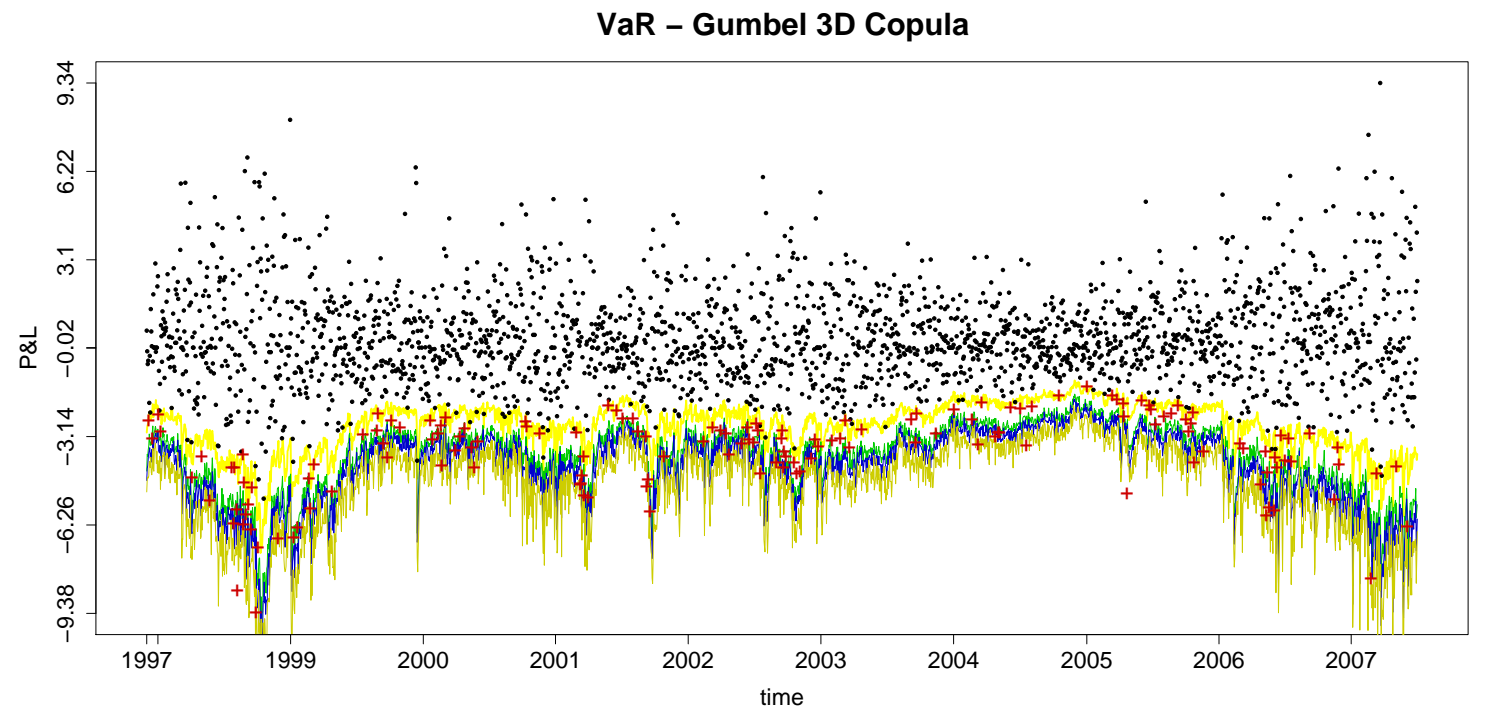

Fig. 9: $\widehat{\operatorname{VaR}}(\alpha)$ and P\&L (dots), estimated with 3-dimensional simple Gumbel copula, $\alpha_{1}=0.05\left(\hat{\alpha}_{1}=0.0612\right), \alpha_{2}=0.01\left(\hat{\alpha}_{2}=0.0232\right), \alpha_{3}=0.005\left(\hat{\alpha}_{3}=0.016\right)$ and $\alpha_{4}=0.001\left(\hat{\alpha}_{4}=0.006\right)$.

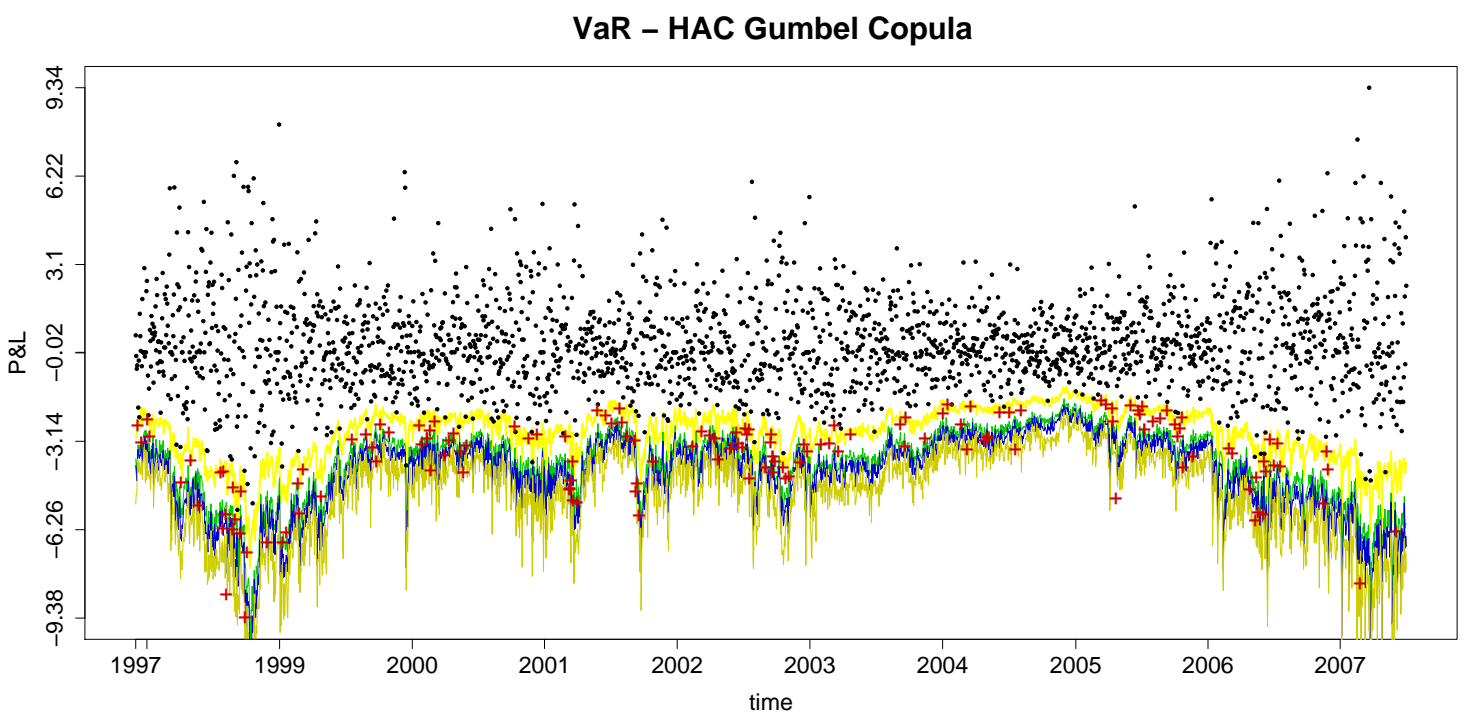

Fig. 10: $\widehat{\operatorname{VaR}}(\alpha)$ and P\&L (dots), estimated with 3-dimensional HAC with Gumbel generators, $\alpha_{1}=0.05\left(\hat{\alpha}_{1}=0.0592\right), \alpha_{2}=0.01\left(\hat{\alpha}_{2}=0.0208\right), \alpha_{3}=0.005\left(\hat{\alpha}_{3}=0.014\right)$ and $\alpha_{4}=0.001\left(\hat{\alpha}_{4}=0.004\right)$. 
Embrechts, P., McNeil, A. and Straumann, D. (1999b). Correlation: Pitfalls and alternatives, RISK May: 69-71.

Frank, M. J. (1979). On the simultaneous associativity of $f(x, y)$ and $x+y-f(x, y)$, Aequationes Mathematicae 19: 194-226.

Frees, E. and Valdez, E. (1998). Understanding relationships using copulas, North American Actuarial Journal 2: 1-125.

Frey, R. and McNeil, A. J. (2003). Dependent defaults in models of portfolio credit risk, Journal of Risk 6(1): 59-92.

Genest, C. and Rivest, L.-P. (1989). A characterization of Gumbel family of extreme value distributions, Statistics and Probability Letters 8: 207-211.

Giacomini, E. and Härdle, W. (2005). Value-at-risk calculations with time varying copulae, Proceedings 55th International Statistical Institute, Sydney 2005.

Gumbel, E. J. (1960). Distributions des valeurs extrêmes en plusieurs dimensions, Publ. Inst. Statist. Univ. Paris 9: 171-173.

Hoeffding, W. (1940). Masstabinvariante Korrelationstheorie, Schriften des Mathematischen Instituts und des Instituts für Angewandte Mathematik der Universität Berlin 5(3): 179-233.

Hoeffding, W. (1941). Masstabinvariante Korrelationsmasse für diskontinuierliche Verteilungen, Archiv für die mathematische Wirtschafts- und Sozialforschung 7: 4970 .

Joe, H. (1997). Multivariate Models and Dependence Concepts, Chapman and Hall, London.

Joe, H. and Xu, J. J. (1996). The estimation method of inference functions for margins for multivariate models, Technical Report 166, Department of Statistics, University of British Columbia.

Marshall, A. W. and Olkin, J. (1988). Families of multivariate distributions, Journal of the American Statistical Association 83: 834-841.

McNeil, A. J. (2008). Sampling nested Archimedean copulas, Journal Statistical Computation and Simulation. forthcoming.

Nelsen, R. B. (2006). An Introduction to Copulas, Springer Verlag, New York.

Okhrin, O., Okhrin, Y. and Schmid, W. (2009a). On the structure and estimation of hierarchical Archimedean copulas. under revision in Journal of Econometrics.

Okhrin, O., Okhrin, Y. and Schmid, W. (2009b). Properties of Hierarchical Archimedean Copulas, SFB 649 Discussion Paper 2009-014, Sonderforschungsbereich 649, Humboldt-Universität zu Berlin, Germany. available at http://sfb649.wiwi.huberlin.de/papers/pdf/SFB649DP2009-014.pdf.

Patton, A. J. (2004). On the out-of-sample importance of skewness and asymmetric dependence for asset allocation, Journal of Financial Econometrics 2: 130-168. 
Savu, C. and Trede, M. (2006). Hierarchical Archimedean copulas, Discussion paper, University of Muenster.

Sklar, A. (1959). Fonctions de répartition à n dimensions et leurs marges, 8: 229-231.

Whelan, N. (2004). Sampling from Archimedean copulas, Quantitative Finance 4: 339352. 


\section{SFB 649 Discussion Paper Series 2009}

For a complete list of Discussion Papers published by the SFB 649, please visit http://sfb649. wiwi. hu-berlin.de.

001 "Implied Market Price of Weather Risk" by Wolfgang Härdle and Brenda López Cabrera, J anuary 2009.

002 "On the Systemic Nature of Weather Risk" by Guenther Filler, Martin Odening, Ostap Okhrin and Wei Xu, January 2009.

003 "Localized Realized Volatility Modelling" by Ying Chen, Wolfgang Karl Härdle and Uta Pigorsch, January 2009.

004 "New recipes for estimating default intensities" by Alexander Baranovski, Carsten von Lieres and André Wilch, January 2009.

005 "Panel Cointegration Testing in the Presence of a Time Trend" by Bernd Droge and Deniz Dilan Karaman Örsal, January 2009.

006 "Regulatory Risk under Optimal Incentive Regulation" by Roland Strausz, January 2009.

007 "Combination of multivariate volatility forecasts" by Alessandra Amendola and Giuseppe Storti, J anuary 2009.

008 "Mortality modeling: Lee-Carter and the macroeconomy" by Katja Hanewald, January 2009.

009 "Stochastic Population Forecast for Germany and its Consequence for the German Pension System" by Wolfgang Härdle and Alena Mysickova, February 2009.

010 "A Microeconomic Explanation of the EPK Paradox" by Wolfgang Härdle, Volker Krätschmer and Rouslan Moro, February 2009.

011 "Defending Against Speculative Attacks" by Tijmen Daniëls, Henk Jager and Franc Klaassen, February 2009.

012 "On the Existence of the Moments of the Asymptotic Trace Statistic" by Deniz Dilan Karaman Örsal and Bernd Droge, February 2009.

013 "CDO Pricing with Copulae" by Barbara Choros, Wolfgang Härdle and Ostap Okhrin, March 2009.

014 "Properties of Hierarchical Archimedean Copulas" by Ostap Okhrin, Yarema Okhrin and Wolfgang Schmid, March 2009.

015 "Stochastic Mortality, Macroeconomic Risks, and Life Insurer Solvency" by Katja Hanewald, Thomas Post and Helmut Gründl, March 2009.

016 "Men, Women, and the Ballot Woman Suffrage in the United States" by Sebastian Braun and Michael Kvasnicka, March 2009.

017 "The Importance of Two-Sided Heterogeneity for the Cyclicality of Labour Market Dynamics" by Ronald Bachmann and Peggy David, March 2009.

018 "Transparency through Financial Claims with Fingerprints - A Free Market Mechanism for Preventing Mortgage Securitization Induced Financial Crises" by Helmut Gründl and Thomas Post, March 2009.

019 "A Joint Analysis of the KOSPI 200 Option and ODAX Option Markets Dynamics" by Ji Cao, Wolfgang Härdle and Julius Mungo, March 2009.

020 "Putting Up a Good Fight: The Galí-Monacelli Model versus 'The Six Major Puzzles in International Macroeconomics'", by Stefan Ried, April 2009.

021 "Spectral estimation of the fractional order of a Lévy process" by Denis Belomestny, April 2009.

022 "Individual Welfare Gains from Deferred Life-Annuities under Stochastic Lee-Carter Mortality" by Thomas Post, April 2009.

\section{SFB 649, Spandauer Straße 1, D-10178 Berlin http:/ / sfb649.wiwi.hu-berlin.de}




\section{SFB 649 Discussion Paper Series 2009}

For a complete list of Discussion Papers published by the SFB 649, please visit http: //sfb649. wiwi.hu-berlin. de.

023 "Pricing Bermudan options using regression: optimal rates of convergence for lower estimates" by Denis Belomestny, April 2009.

024 "Incorporating the Dynamics of Leverage into Default Prediction" by Gunter Löffler and Alina Maurer, April 2009.

025 "Measuring the effects of geographical distance on stock market correlation" by Stefanie Eckel, Gunter Löffler, Alina Maurer and Volker Schmidt, April 2009.

026 "Regression methods for stochastic control problems and their convergence analysis" by Denis Belomestny, Anastasia Kolodko and J ohn Schoenmakers, May 2009.

027 "Unionisation Structures, Productivity, and Firm Performance" by Sebastian Braun, May 2009.

028 "Optimal Smoothing for a Computationally and Statistically Efficient Single Index Estimator" by Yingcun Xia, Wolfgang Härdle and Oliver Linton, May 2009.

029 "Controllability and Persistence of Money Market Rates along the Yield Curve: Evidence from the Euro Area" by Ulrike Busch and Dieter Nautz, May 2009.

030 "Non-constant Hazard Function and Inflation Dynamics" by Fang Yao, May 2009.

031 "De copulis non est disputandum - Copulae: An Overview" by Wolfgang Härdle and Ostap Okhrin, May 2009. 